\title{
L'activité narrative dans ses dimensions multi instrumentée et créative en situation pédagogique
}

Narrative activity, mediated and creative dimensions in educational situations

\section{Françoise Decortis}

\section{(2) OpenEdition}

\section{Journals}

Édition électronique

URL : http://journals.openedition.org/activites/520

DOI : 10.4000 /activites.520

ISSN : $1765-2723$

Éditeur

ARPACT - Association Recherches et Pratiques sur les ACTivités

Référence électronique

Françoise Decortis, «L'activité narrative dans ses dimensions multi instrumentée et créative en situation pédagogique », Activités [En ligne], 10-1 | Avril 2013, mis en ligne le 15 avril 2013, consulté le 19 avril 2019. URL : http://journals.openedition.org/activites/520 ; DOI : 10.4000/activites.520

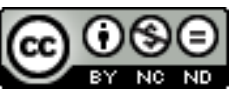

Activités est mis à disposition selon les termes de la licence Creative Commons Attribution - Pas d'Utilisation Commerciale - Pas de Modification 4.0 International. 


\title{
L'activité narrative dans ses dimensions multi instrumentée et créative en situation pédagogique
}

\author{
Françoise Decortis \\ Laboratoire Paragraphe, équipe C3U - Université Paris 8 - 2 rue de la Liberté \\ 93200 Saint Denis \\ Francoise.decortis@univ-paris8.fr
}

\begin{abstract}
Narrative activity, mediated and creative dimensions in educational situations. Narrative activity is an activity to which insufficient attention has been paid in the literature so far although in ergonomics, many studies are beginning to recognize its importance. Our study examines children's narrative activity guided by adults in a pedagogical situation, mobilizing in a complementary manner concepts from Rabardel's instrumental theory and Vygotski's theory of creative imagination. These theoretical frameworks allow us to account for the mediated and creative aspects of narrative activity. Based on analyses of educational situations and on these concepts, a model of narrative activity (NAM) is proposed and illustrated. It includes four main phases: exploration, inspiration, production and sharing. Narrative activity is a way for subjects to develop their creative resources. To contribute to this, teachers operate at two levels. On the one hand, they help children to operate instrumental geneses at functional and semantic levels. Several types of activity (sensory, descriptive and manipulative) contribute to this. On the other hand, teachers use various media to commit children to constituting a kind of "internal laboratory". Children are invited to think about variations of tools with which they experiment and to listen to the echos which such variations create in them. These reflexive interpersonal mediations consist in opening the subject's palette of resources, in widening their possibilities for dissociation and association, and in generating materials which will be useful for their narrative production.
\end{abstract}

\section{KEYWORDS}

Narrative activity, instruments, mediations, educational situations, creativity.

\section{Introduction}

L'activité narrative forme un registre de l'activité sans doute plus important que l'attention que l'on a bien voulu lui donner jusqu'ici. Son importance commence à être reconnue dans bon nombre d'études en ergonomie (Bationo-Tillon, 2006; Beaujouan, 2011 ; Decortis, 2008 ; Decortis, \& Bationo-Tillon (sous presse) ; Decortis \& Rizzo, 2002) ; Decortis, Rizzo, Daele, Polazzi, \& Saudelli, 2001 ; Decortis, Rizoo, \& Saudelli (2003); Marchand, 2011). Dans les entreprises, les récits expérientiels (Marchand, 2011 ; Marchand \& Falzon, 2011), sont utilisés au sein de collectifs et sont estimés comme étant d'une grande utilité dans la gestion des risques. Dans une étude récente, Beaujouan et Daniellou (2012) cherchent à comprendre les apports et les limites des récits professionnels à l'apprentissage d'un métier. 
À l'école, le récit peut être utilisé pour aider les élèves à revisiter les étapes qui leur ont permis de découvrir la solution à un problème. Sa modélisation est déterminante pour concevoir des technologies narratives (Annany et Cassell, 2001 ; Cassell \& Ryokai, 2001 ; Decortis, Rizzo, Daele, Polazzi, \& Saudelli, 2001 ; Guha, Druin, Montemayor, Chipman \& Farber, 2007 ; Raffle, Vaucelle, Wang, \& Ishii, 2007 ; Ryokai \& Cassell, 1999 ; Vaucelle, 2010).

Notre approche de l'activité narrative concerne des enfants guidés par des adultes en situation pédagogique. Notre objectif est de rendre compte des dimensions multi instrumentée et créative de l'activité narrative qui, jusqu'ici sont restées dans l'ombre. Pour éclairer ces dimensions, nous mobilisons de façon complémentaire l'approche instrumentale (Rabardel, 1995) et le cycle de l'activité créatrice de l'imagination (Vygotski, 1930/1983).

Dans ce texte, nous progressons en trois temps. Dans un premier temps, nous explicitons nos choix théoriques pour définir et étudier l'activité narrative. Dans un deuxième temps, nous présentons une situation pédagogique, le «laboratoire des rythmes », menée dans une classe avec des enfants qui ont entre 6 et 7 ans. Dans un troisième temps, nous présentons le modèle NAM («Narrative Activity Model», Decortis, 2008) et ses quatre phases en lien avec cette situation pédagogique et quand cela est pertinent nous élargissons à d'autres situations pédagogiques. Enfin, nous concluons en discutant de la contribution de cette recherche à la compréhension du développement des ressources créatives et narratives et de la recherche de nouvelles perspectives.

\section{1.- L'activité narrative : dimensions instrumentale et créative}

Commençons par donner une définition de ce que nous entendons ici quand nous parlons de «l'activité narrative». Premièrement, nous considérons l'activité narrative comme une activité instrumentée et créative, produisant quelque chose qui peut être un récit ou une histoire, que le sujet construit en puisant dans son expérience présente ou passée. Deuxièmement, le récit ou l'histoire peut prendre une forme écrite ou orale, mais pas seulement, il peut prendre une forme iconique ou corporelle, ou toutes autres formes significatives pour le sujet. Troisièmement, l'activité narrative peut être construite, au moins partiellement, par le sujet en situation et peut évoluer sous son action, progressivement, en fonction des situations concrètes vécues et de la diversité de moyens et de ressources auxquels le sujet recourt. Les situations vécues, présentes ou passées, constituent des sources d'inspiration, fournissent des matériaux pour le sujet, car le récit ou l'histoire peut être un compte rendu de l'expérience, il peut rapporter un épisode marquant d'une situation singulière, mais il peut être aussi imaginaire. Les moments de partage de l'histoire constituent une étape importante de l'activité narrative.

Dans cet article, nous allons nous focaliser sur une situation pédagogique observée à l'école. Cependant, nous pensons que cette approche de l'activité narrative peut s'étendre à d'autres situations dans le travail, la vie quotidienne et la médiation culturelle. Nous allons maintenant apporter des éléments de précision à cette définition en examinant d'un peu plus près l'activité narrative en situation pédagogique, sa dimension instrumentale et enfin sa dimension créative.

\section{1.- Activité narrative et situations pédagogiques}

Bruner (1996) conçoit le récit en tant que mode de pensée, expression de la manière dont une culture considère le monde et véhicule de l'élaboration des significations. C'est au travers de nos récits que nous construisons une conception de ce que nous sommes et c'est au travers des récits qu'une culture fournit à ses membres des modèles d'identité et d'action. Ainsi, Bruner souligne la centralité du mode narratif dans la construction de l'identité et le rôle que l'école doit jouer. L'école doit « le cultiver, le nourrir, cesser de le prendre pour une chose acquise ». Plus loin, Bruner poursuit «utiliser les récits comme un instrument de l'esprit demande que nous y travaillions, que nous en lisions, que nous en fabriquions, que nous les 
analysions, que nous comprenions l'art de les construire, que nous en percevions les usages, que nous en discutions ». Or comme Bruner le déplore (1996), dans la plupart des écoles, les arts du récit (chant, drame, fiction, théâtre) relèvent davantage de la décoration que de la nécessité, ils appartiennent au domaine du loisir. Pourtant, nous donnons une forme narrative à la manière dont nous rendons compte de nos origines culturelles et de nos croyances les plus chères. Notre expérience immédiate, ce qui est arrivé aujourd'hui ou hier, tout cela est mis en forme au travers de récits. De manière plus frappante, c'est sous cette forme que nous représentons notre existence à nous-mêmes aussi bien qu'aux autres.

Bruner souligne encore que notre tradition pédagogique rend peu justice à l'importance de l'intersubjectivité et ce, tant en ce qui concerne la posture de l'enseignant, omniscient, qui transmet à des apprenants censés ne rien connaître que des apprenants entre eux. Ce que Bruner nomme «l'apprentissage mutuel », forme à des manières de faire et de penser, constitue un processus interactif qui développe l'échange intersubjectif. Ce qui revient à reconnaître le point de vue de l'enfant, à encourager chacun à exprimer ses propres vues, à nourrir la compréhension par la discussion et la coopération. Comme il le souligne, «l'enfant n'est pas vraiment ignorant et n'est pas un récipient vide, mais c'est quelqu'un qui est capable de raisonner, de donner du sens, par lui-même et au travers de la discussion avec les autres ».

Voyons maintenant ce que nous considérons être la spécificité de notre approche autour de deux axes principaux : la dimension instrumentée de l'activité narrative et la dimension créative de celle-ci.

\section{2.- L'activité narrative instrumentée}

Dans sa proposition d'une théorie instrumentale étendue, Rabardel (1999) souligne l'importance d'envisager l'activité humaine sous l'angle de la diversité de ses moyens, c'està-dire l'ensemble des instruments, matériels, psychologiques, internes ou externes aux sujets, que ces derniers vont élaborer et mobiliser au sein de l'activité. L'instrument matériel, « objet de forme particulière aux propriétés physiques déterminées », permet de transformer un objet matériel avec un outil qui est manipulé par le sujet. L'instrument psychologique, quant à lui, distingué des premiers par la direction de leur action vers le psychisme, permet de gérer sa propre activité.

Après avoir rappelé que le langage peut être considéré en tant qu'instrument, Rabardel (1999) soutient qu'instrument technique et instrument psychologique sont solidaires. Le langage peut, en effet, se concevoir comme un véritable instrument dans le sens où il est un moyen d'agir sur l'activité humaine pour la réorganiser par sa fonction d'action et de contrôle.

Dans la situation que nous étudions ici, le langage coexiste avec un ensemble d'instruments et de ressources matérielles et psychologiques tels que la production de traces écrites, la production de sons, la lecture, le dessin et le mime. En effet, nous devons parler d'instruments au pluriel, car à l'école, alors que la plupart des activités narratives sont centrées sur la production écrite et verbale, Ackermann (2001) rappelle que les enseignants savent que l'activité narrative est souvent enrichie par un ensemble de médias tels que le dessin, la musique, l'expression corporelle. Malaguzzi (1987) a lui bien décrit la façon dont les enfants apprennent à s'exprimer dans des centaines de langages.

L'approche instrumentale permet de comprendre et de spécifier la nature des médiations des situations où des instruments sont mobilisés. L'instrument est un moyen qui permet la connaissance de l'objet et ses propriétés (médiations épistémiques), l'instrument est le moyen d'une action transformatrice de l'objet (médiations pragmatiques), le sujet apprend à se connaître, à se gérer, à se transformer (médiations réflexives), l'instrument médiatise les relations à autrui (médiations interpersonnelles), ces dernières pouvant être épistémiques ou pragmatiques, selon qu'il s'agit de connaître les autres ou d'agir (Folcher \& Rabardel, 2004).

Lorsqu'elle est matérialisée sur un support (dans un cahier par exemple), une histoire écrite 
par un enfant peut constituer une trace pour les autres. Communiquée à d'autres, elle devient un matériel partagé et partageable qui pourra être poursuivi et approprié par d'autres. Même si souvent une histoire est créée par chaque enfant individuellement, dans de nombreuses situations, le collectif est important. Le pôle du collectif est doublement constitué : ceux à qui un enfant raconte une histoire, ceux avec qui on peut créer un récit ou une histoire, par qui on peut être aidé (d'autres enfants, l'enseignant). Un récit est toujours adressé à un autre (un enfant, un parent, l'enseignant), à soi (on se raconte notre ou une histoire). En fonction de l'audience, l'enfant ajuste son récit, il raconte à celui qui sait écouter (Bruner,1996).

La dimension coopérative peut revêtir diverses formes par exemple les enfants créent ensemble une histoire en décidant des personnages, des événements et des actions ; ou l'histoire se construit en chaîne par ajout progressif de fragments. Ainsi aux rapports entre l'enfant et les instruments, viennent s'ajouter les interactions du sujet avec les autres par le biais de coopérations. Nous référant à la théorie instrumentale, les instruments partagés remplissent une fonction de médiation collaborative.

L'activité a deux types d'orientation, productive, la réalisation de tâches, et constructive, l'élaboration de ressources internes et externes, c'est-à-dire les instruments, compétences, schèmes et systèmes de valeurs. L'activité constructive permet de produire les conditions et les moyens de l'activité future (Rabardel, 2005). Deux unités d'analyse sont mises en relation avec ces deux orientations, l'activité médiatisée d'une part et l'appropriation des outils culturels, des usages et du développement des instruments d'autre part. Cette approche permet ainsi de comprendre non seulement la nature des activités dans l'usage des artefacts, mais aussi les modalités de développement des personnes par le biais des processus d'appropriation, notamment le développement des ressources pour l'action et des compétences (Folcher \& Rabardel, 2004).

Considérant que l'instrument n'est pas donné d'emblée aux utilisateurs, une approche développementale de l'appropriation des outils est étudiée sous l'angle de ce que Rabardel (1995) appelle la «genèse instrumentale », doublement orientée : vers le sujet d'une part, émergence et évolution des schèmes d'utilisation (il s'agit de l'instrumentation), et vers l'artefact, d'autre part, par l'enrichissement de ses propriétés et de ses spécifications par le sujet (il s'agit de l'instrumentalisation).

Enfin, dernier point qui nous aidera à faire le lien avec la dimension créative de l'activité, Rabardel et Béguin (2005) soulignent que «l'esprit d'invention» de l'utilisateur et la créativité constituent des caractéristiques ontologiques de l'usage de l'instrument, des processus d'appropriation des artefacts et plus généralement de l'activité en situation. Le sujet qui fait preuve de créativité cherche à exploiter les ressources disponibles dans l'environnement de son activité et à les inscrire au service de son action. Nous allons maintenant envisager la question de la dimension créative de l'activité narrative en nous appuyant sur la contribution de Vygotski.

\section{3.- La dimension créative de l'activité narrative}

Rendre compte des dimensions créatives de l'activité narrative est essentiel, à la fois sur un plan empirique et théorique. Car si les enfants développent leurs capacités langagières et narratives dès l'âge de 5 ans, c'est également une période où l'imagination et la créativité sont importantes. Pour Vygotski (1930/1983) par le jeu ou par le récit, qui ne sont pas de simples reproductions d'impressions vécues, mais des réélaborations créatrices de celles-ci, l'enfant met en relation et assemble les données de l'expérience pour construire une nouvelle réalité répondant à ses curiosités et à ses besoins. Dans la mesure où l'imagination se construit avec des matériaux pris dans la réalité, pour nourrir son imagination, il faut que l'enfant puisse grandir dans un milieu riche en impulsions et en stimulations. Vygotski (1930/1983) n'oppose donc pas réalité et imagination, mais explore quatre types de relations (Figure 1). La première relation indique que l'activité créative de l'imagination dépend directement de la richesse et de la variété des expériences, car les constructions de la fantaisie sont composées du matériel fourni par l'expérience. La fantaisie n'est pas 
antithétique de la mémoire d'événements vécus, mais elle est soutenue par elle. La fantaisie retravaille les traces des événements dans des formes nouvelles. La deuxième relation est possible grâce à l'expérience sociale. Certains événements dont chacun peut parler n'ont pas nécessairement été vécus en première personne, pourtant nous pouvons en parler, car nous en avons des représentations construites à travers nos interactions avec les autres. La troisième relation est émotionnelle. La loi du signe émotionnel commun et la loi de la réalité de l'imagination décrivent le fait que les images de la fantaisie fournissent un langage à nos sensations. Les éléments observés sont mis en relation selon notre humeur plutôt que par des relations logiques. D'autre part, chaque forme d'imagination créative comprend des éléments affectifs. La loi de la réalité de l'imagination offre une passerelle vers la quatrième relation qui unit réalité et imagination. Une fois qu'un produit de l'imagination est concrètement incarné dans quelque chose, il commence à exister en tant que tel. Ce qui peut être vrai pour des objets physiques ou pour des idées et des émotions.

Ainsi, pour Vygotski (1930/1983), les produits de l'imagination ont une longue histoire et leur développement suit un chemin circulaire : les éléments à partir desquels ils ont été construits sont issus de la réalité, ils sont ensuite retravaillés par celui qui crée avant de retourner à la réalité pour constituer de nouvelles forces actives, transformatrices de cette réalité. Ceci correspond au cycle complet de l'opération créative de l'imagination. En première approche nous considérons que le cycle de l'activité créatrice de l'imagination nous offre un cadrage pour comprendre la façon dont l'activité narrative se construit dans un empan temporel large, de l'échelle de la vie à des moments bien délimités dans le temps. Dans cette perspective nous considérons d'une part qu'aider l'enfant à compléter le cycle de l'activité créatrice de l'imagination qui démarre de la connaissance sensorielle de la réalité et retourne à la réalité par une modification active de l'environnement produit par l'incarnation de l'imagination est essentiel. D'autre part, ce processus de corporéité concerne à la fois les aspects matériels et techniques, mais également les aspects émotionnels.

Vygotski (1930/1983) identifie deux mécanismes de l'imagination: l'expérience et la réélaboration de l'expérience. Le point d'appui de la créativité est marqué par des perceptions externes et internes, sédiments de notre expérience. Ainsi ce que l'enfant voit ou entend, constitue le premier point d'appui pour sa créativité à venir. Il accumule ainsi le matériel à partir duquel, ensuite, sa fantaisie se construira. Vient ensuite un processus complexe de réélaboration du matériel qui comprend deux parties essentielles la dissociation et l'association des impressions reçues. Chaque impression constitue un tout complexe, qui est le résultat d'une quantité de parties distinctes. La dissociation consiste à séparer ce tout complexe en ses parties: certaines sont mises en évidence et conservées, d'autres sont abandonnées. L'association, quant à elle vise la réunion des éléments dissociés et transformés. L'association peut avoir plusieurs formes entre une association purement subjective des images ou une autre plus objective. Selon Vygotski (1930/1983), le moment conclusif du travail préparatoire de l'imagination est celui d'assembler les images isolées, les organiser en systèmes, construire un cadre d'ensemble. L'activité créatrice de l'imagination n'est cependant pas terminée. Le cycle ne sera complet que quand l'imagination sera incarnée, ou cristallisée, dans des images extériorisées. 


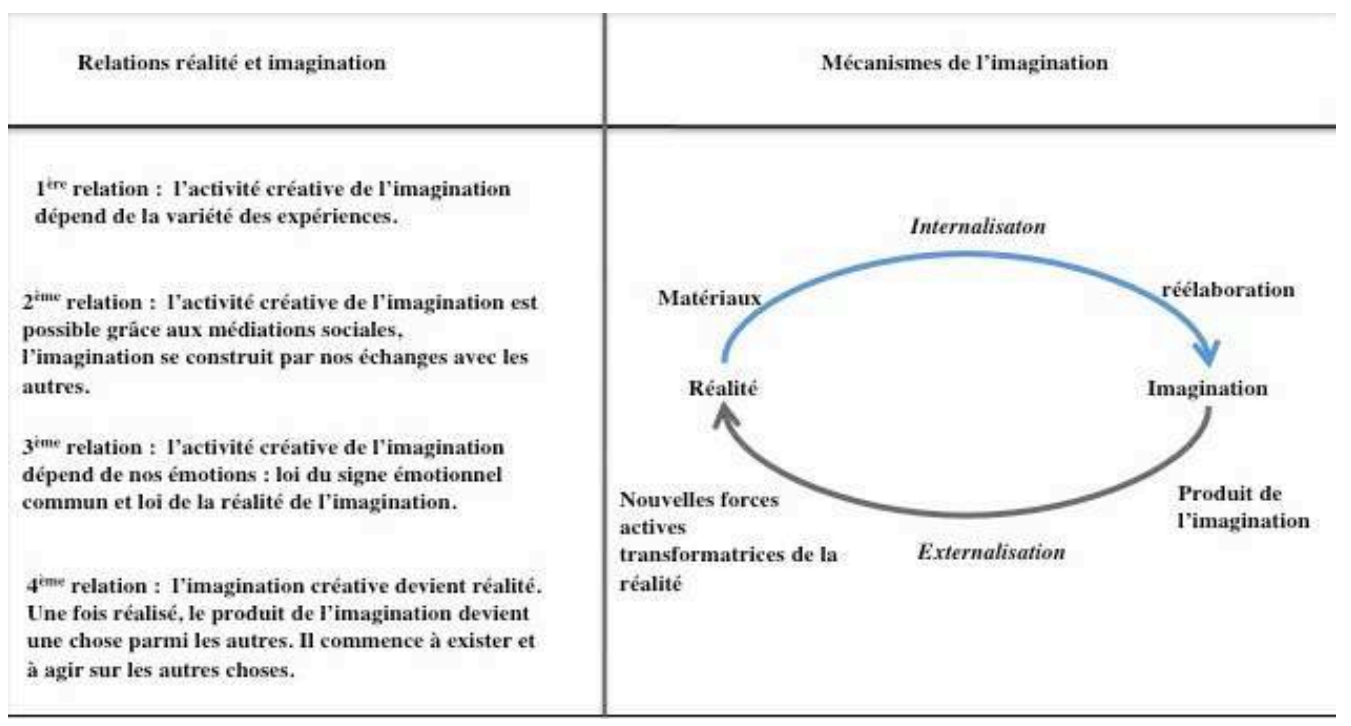

Figure 1 : Relations entre réalité et imagination et mécanismes de l'imagination (Vygotski, 1930/1983)

Figure 1: Relationships between reality and imagination and mechanisms of the imagination (Vygotski, 1930/1983)

\section{2.- Étude d'une situation pédagogique : le « laboratoire des rythmes »}

Parmi plusieurs situations pédagogiques étudiées (Decortis, 2008), nous choisissons, dans cet article, d'analyser l'une d'elles, le «laboratoire des rythmes». Dans cette situation, les enfants sont invités à créer des histoires à partir de l'exploration de sons et de rythmes. Les enfants construisent des instruments musicaux à partir de matériaux de récupération et d'objets chinés dans le jardin (ex. : bouteille en plastique, morceaux de bois, marron, etc.). Ensuite, utilisant ceux-ci et d'autres instruments musicaux (tambourins, clochettes, triangles, etc.), ils explorent des sons et des rythmes. Enfin, ils produisent des histoires par petits groupes et les partagent.

\section{1.- Repères méthodologiques}

La méthodologie repose sur des observations dans une école de l'enseignement primaire. Le cadre scolaire crée, comme le souligne Vergnaud (2002), des conditions en vue de faire surgir des observables qui se prêtent à leur analyse in situ. Cette démarche s'appuie sur une analyse de situations pédagogiques qui impliquent, pour chaque situation observée, un enseignant et des enfants.

Dans cet article, nous reportons nos observations du laboratoire des rythmes dans une classe d'enfants qui ont entre 6 et 7 ans. La méthodologie de récolte de données a été celle de l'observation, l'usage de la vidéo (caméra fixe et caméra mobile) pour récolter des traces de l'activité.

Concernant le laboratoire des rythmes, nous analysons des données issues de ce dispositif méthodologique : observations ouvertes et systématiques et verbalisations essentiellement. Les échanges entre enfants et avec l'enseignant ont été enregistrés, transcrits et analysés.

\section{2.- Éléments de compréhension de la situation pédagogique}

Le « laboratoire des rythmes » s'est étalé sur deux séances d'une durée totale de trois heures. Si l'on considère les deux séances comme un continuum, on peut identifier dans l'activité observée quatre niveaux successifs, chacun traversé par la découverte des sons et du rythme (Figure 2). Le premier niveau est celui de la fabrication des instruments musicaux. Il couvre 
la presque totalité de la première séance du laboratoire et est réalisé par chaque enfant individuellement. Il consiste à «chiner» des objets susceptibles de contribuer à la fabrication d'instruments musicaux et fabriquer ceux-ci. Le deuxième niveau est celui des prémisses de la construction narrative, il est réalisé par les enfants en petits groupes. Dans le troisième niveau, la création des histoires, les enfants élaborent des bouts de récits en petits groupes en associant sons et fragments narratifs. Dans le quatrième et dernier niveau de l'activité, les histoires sont partagées avec l'ensemble de la classe, chaque groupe présente son histoire aux autres enfants.

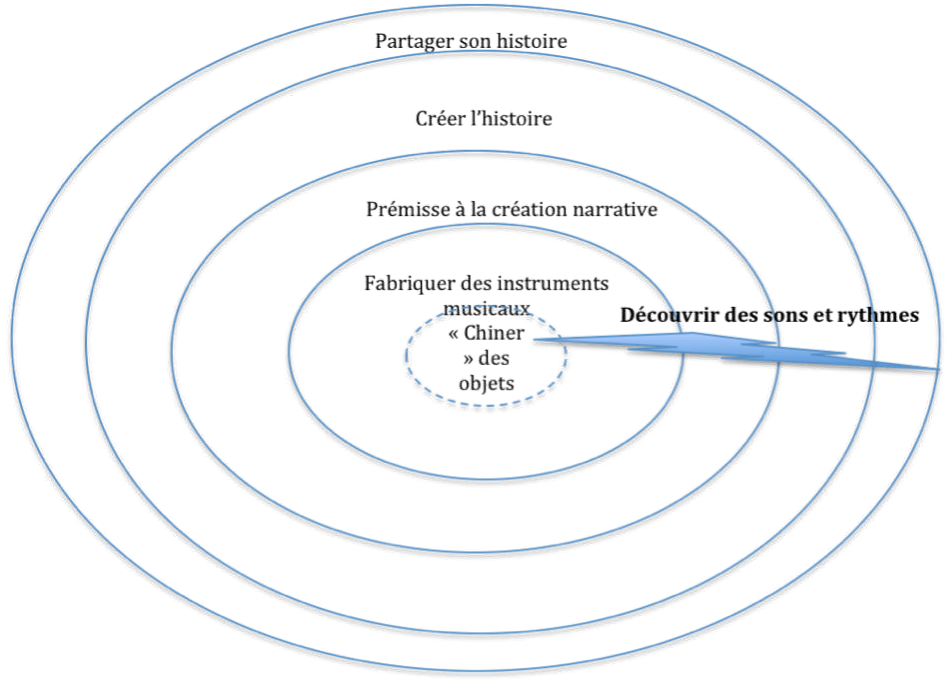

Figure $2:$ Le laboratoire des rythmes

Figure 2: The rhythm laboratory

Cette description sommaire de l'activité laisse entrevoir sa complexité puisqu'elle intègre de la manipulation et de la construction, de l'exploration et de la découverte, de la production narrative et de la «performance » entendue dans le sens de la réalisation d'actes de parole joués et partagés. Avec diverses incitations, l'enseignante désigne le parcours de niveaux successifs de cette expérience.

Au début de la première séance (Figure 3), l'enseignante présente les objectifs de la situation pédagogique: transformer des bouteilles en instruments musicaux en collectant des matériaux pour les remplir, décorer ces instruments, pour ensuite, dans un deuxième temps, créer des histoires.

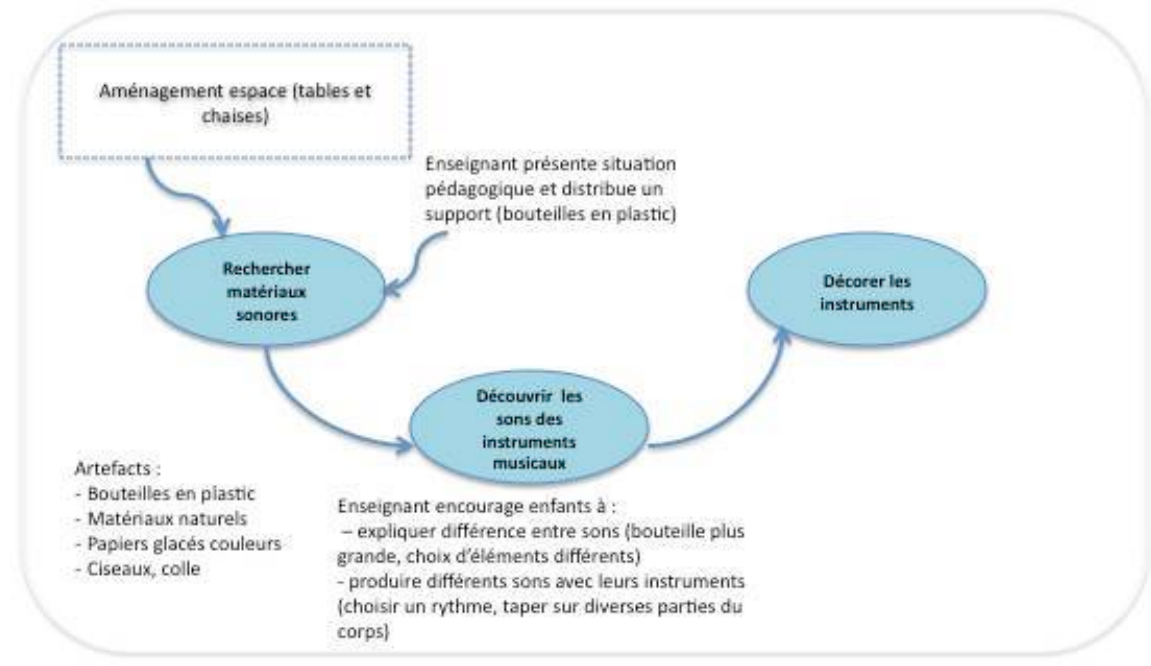

Figure 3 : Première séance du laboratoire des rythmes

Figure 3: First rhythm laboratory session 
$\mathrm{Au}$ début de la deuxième séance, les enfants discutent en petits groupes, regardent et secouent leurs bouteilles. Au fond de la classe, deux tables l'une à côté de l'autre forment une surface de travail qui vont servir aux enfants pour poursuivre leurs explorations (Figure 4).

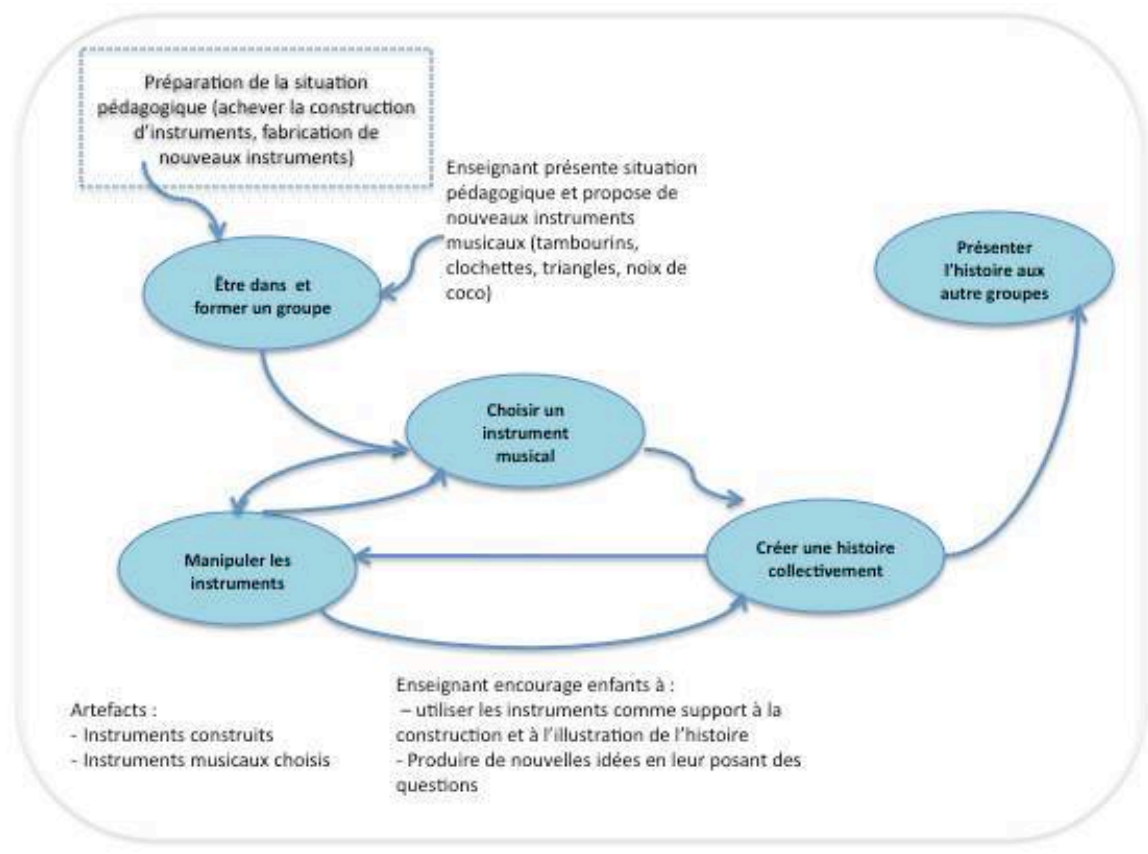

Figure 4 : Deuxième séance du laboratoire des rythmes

Figure 4: Second rhythm laboratory session

On peut maintenant examiner d'un peu plus près ce qui s'y joue.

\section{3.- Éléments d'analyse de l'activité}

Dans cette partie du texte, nous reprenons les éléments présents dans les figures 3 et 4 en les décrivant de façon chronologique tels qu'ils sont apparus dans le cours de la situation pédagogique.

\subsection{1.- Première séance \\ Fabriquer des instruments musicaux}

Au début de la première séance du «laboratoire des rythmes », l'enseignante a apporté des bouteilles en plastique, certaines de $50 \mathrm{cl}$ et d'autres plus grandes d'un litre. Elle commence par changer la disposition de la classe : tables et chaises sont déplacées contre une paroi pour laisser un espace de travail ample et ouvert. Les enfants se réunissent autour d'elle. Elle distribue les bouteilles et présente les objectifs de la situation pédagogique : transformer ces artefacts en instruments musicaux en collectant des matériaux pour les remplir, décorer ces instruments, pour ensuite, dans un deuxième temps, créer des histoires.

\section{Extrait 1 :}

Enseignante (E.) : qui va me rappeler ce qu'on va aller faire? Sarah qu'est-ce qu'on va faire?

Sarah : on va aller dehors et chercher des choses qui peuvent aller dedans... donc pas trop grandes et après on fermera avec le bouchon, et on fera de la musique et ce sera un instrument... et après on prendra de la colle et on le décorera, on décorera les bouteilles avec du papier.

Un enfant commence à faire des bruits en pressant sa bouteille. 


\section{Extrait 2 :}

Enseignante (E.) : "rien que ça...fais-le, fais-le. Regardez, elle disait « ça déjà ça fait de la musique ».

E. : « vous n'avez encore rien mis dedans et vous êtes déjà capable de faire de la musique. Donc on apportera d'autres bouteilles vides pour que vous puissiez explorer d'autres sonorités ».

Les enfants s'éparpillent dans le jardin à la recherche de matériaux pour remplir leurs bouteilles : ils récoltent des cailloux, bâtons de bois de différentes dimensions, fruits secs, etc. Dans le jardin, formant un cercle autour de l'enseignant, les enfants commencent à confronter les sons produits par leurs instruments musicaux.

\section{Extrait 3 :}

E. : alors on va le faire chacun à son tour, pour pouvoir entendre la différence, on va se mettre en cercle

E. (s'adressant à un enfant) : alors fait le d'abord

L'enfant à sa droite secoue rythmiquement son instrument en le saisissant par l'extrémité du bouchon

E. : ça va... à toi maintenant (en s'adressant à un deuxième enfant),

L'enfant bouge la bouteille de la même façon, mais à un rythme plus lent.

$\mathrm{E}$ : vous entendez déjà la différence entre les deux... (l'enfant continue plus vite)

E. : qu'est-ce que tu as dedans toi?

Enft. : moi, j'ai des...j'ai des cailloux

De retour en classe, les enfants reprennent une disposition circulaire, jouent chacun à leur tour de leur instrument, continuent d'explorer des sons, certains en le saisissant par le bouchon, d'autres en prenant la bouteille des deux mains, d'autres encore tiennent la bouteille d'une main et la tapent contre l'autre.

Ils sont invités à décrire le contenu de leur instrument pour expliquer les différences de son. Dans l'extrait suivant, l'enseignante demande à certains enfants de décrire le contenu de leur instrument pour expliquer les différences de son.

\section{Extrait 4 :}

E. : elle a un bruit très différent, attends... Alexandra, tu as pu expliquer tout à l'heure en montant, tu as expliqué pourquoi toi, chez toi c'est un bruit très très différent

Sarah : parce qu'elle a une grande bouteille et puis...

Alexandra : c'est un grand bâton

E. : elle a mis un très gros et grand bâton dedans, vous avez vu? et quand le gros bâton tape contre la paroi de la bouteille, ça fait un son très différent. Refais-le qu'on entende bien. Vas-y Raphaël... vous entendez que cela fait des sons très différents...

Dans l'extrait suivant, un enfant propose de jouer tous ensemble, mais l'enseignante renvoie ce moment à plus tard. Elle invite des petits groupes de trois et quatre enfants à secouer leurs bouteilles en même temps.

\section{Extrait 5 :}

Alexandra : eh tous ensemble!

E. : attends, attends, attends... Certains enfants commencent à jouer

E. : stop stop stop... alors maintenant je vais demander à Shanaël, Maud et Alexandra de le faire ensemble (les trois enfants jouent en même temps).

E. : merci à Gilles, Rapahël et Caroline, les enfants jouent en même temps

E. stop Gary, Sarah... Marie tu l'as fait?

Marie : non

E. : Marie et Eva (les quatre enfants jouent en même temps)

E. : et maintenant tous ensemble. Tous les enfants jouent en même temps. Gary commence à bouger en suivant le rythme, Shanaël l'imite.

Ensuite, en suivant l'exemple de l'enseignante, les enfants expérimentent différentes façons de produire des sons: en les tapant sur une partie du corps (tête, épaules, genoux), en 
changeant le rythme, en donnant des coups plus ou moins forts, etc.

\section{Extrait 6 :}

E. : et maintenant on va essayer peut être pas de seulement de faire comme ça (elle secoue la bouteille en saisissant le bouchon), on va peut être essayer en tapant comme ça (elle tape sur la tête, puis sur le genou et sur le coude), à des endroits différents (les enfants l'imitent)

Voilà très bien...

Shanaël : moi sur mon épaule

E. : vas-y! (Shanaël tape la bouteille sur son épaule)

E. : voilà qui a essayé autre chose?

Les enfants tapent sur les différentes parties de leur corps

E. : oui... sur la tête... oui sur la cuisse, oui... d'accord...

À l'issue de cette découverte des sons, l'enseignante invite les enfants à s'asseoir par terre, en cercle. Elle place au centre des feuilles de papier coloré, de la colle et des ciseaux et explique aux enfants comment les utiliser pour la décoration.

\section{Extrait 7 :}

E. : alors il y a plusieurs moyens. Soit je prends des ciseaux et je découpe des petites formes que j'ai décidées, soit je peux même prendre tous les petits morceaux que je déchire (ne les prenez pas trop petits parce que sinon ça met beaucoup de temps, mais ne prenez pas de très grands parce que sinon le résultat n'est pas très joli... et le but est d'aller coller tous les petits bouts... et je vais le plus possible couvrir ma bouteille (...)

La semaine dernière, les enfants qui en ont fait avec moi, il y a certains enfants qui n'ont que recouvrir et il y en a d'autres qui par contre on fait des petits paysages, un petit bateau avec la mer et le soleil... vous faites comme vous voulez, l'important est que vous fassiez votre instrument à vous comme vous en avez envie; d'accord?

L'enseignant recommande de ne pas se disputer pour le matériel. Les enfants commencent à travailler. Quelques minutes après, l'enseignante met un disque de Carmina Burana. Elle invite à écouter les percussions et le rythme.

\section{Extrait 8 :}

E. : je vous mets un petit morceau qui s'appelle "Carmina Burana» qui a été composé par un monsieur qui s'appelle Carl Orf. Ce monsieur a aussi inventé une méthode d'apprentissage de la musique avec justement beaucoup de percussions.

Les enfants travaillent avec le fond musical pendant une demi-heure. Certains commentent la musique ou le travail de décoration.

Caroline : c'est une drôle de langue

E. : c'est du latin

Alexandra : on dirait le bossu de Notre Dame (rire)

E. : si vous écoutez même la manière dont ils chantent, c'est, c'est du rythme

Shanaël : moi je trouve que ça, que la musique ressemble un peut aussi à Notre Dame de Paris.

E. : mais c'est ce qu'elle avait dit aussi, mais ce n'est pas la musique de Notre Dame.

L'ensemble des enfants décore les bouteilles dans un style «collage », aucun ne fait de petits paysages. À la fin de la séance, les enfants rangent la pièce.

\subsection{2.- Deuxième séance}

$\mathrm{Au}$ début de la deuxième séance, les enfants sont debout et éparpillés dans la classe. Ils discutent en petits groupes, regardent et secouent leurs bouteilles. Tables et chaises sont dans leur position habituelle (en rang). Au fond de la pièce, deux tables côte à côte, forment une surface de travail plus ample. L'enseignante demande aux enfants de s'asseoir en indiquant que les premières dix minutes seront dédiées à l'achèvement des instruments musicaux et qu'ensuite il faudra inventer une histoire. À une table, un enfant explique à un autre, absent lors de la première séance, comment construire sa bouteille.

Extrait 9:

Raphaël : et donc, il y en a qui ont mis des cailloux (...) 
Léo : on doit mettre des cailloux? moi je préfère des bâtons en bois

Raphaël : oui, mais tu peux mettre autre chose, mais normalement, ça fait de la musique, ça doit faire de la musique, et puis après tu essayes (il secoue la bouteille) pour que ça fasse de la musique et quand...

Léo : tout le monde a pris des trucs de la nature?

Raphaël: oui et après, quand tu fais un son que tu aimes bien, tu reviens et tu vas la décorer, tu la décores avec du papier.

L'enseignante a apporté d'autres instruments (triangle, tambourin, cymbale, noix de coco) qu'elle dépose sur une table au centre de la pièce. Deux enfants remplissent une bouteille vide avec de l'eau pour en faire un autre instrument musical. L'enseignante montre comment faire des bulles avec la bouteille remplie d'eau. Elle invite ensuite les enfants à s'asseoir autour de la grande table.

\section{Extrait 10 :}

E. : voilà ce que je vais vous demander c'est de vous mettre par équipe de deux ou trois. D'accord? (les enfants commencent à se regrouper). Deux ou trois et on ne laisse pas quelqu'un tout seul. Je vois que Gilles est tout seul.

Sarah : Gilles, tu viens avec nous?

E. : donc on va avoir une (elle indique les groupes d'enfants), deux, trois, quatre équipes. Chaque équipe va utiliser les instruments que vous avez fabriqués et va venir choisir ici sur la table un instrument musical en plus parmi ceux que j'ai apportés. D'accord?

E. : Quand vous avez chacun votre bouteille et votre instrument, vous allez vous asseoir à des tables différentes par équipe et là vous allez vous inventer une histoire, mais vous allez pour la raconter, utiliser les instruments de musique pour faire le bruitage.

Extrait 11:

E. : Donc ce que je vous conseille c'est d'abord d'écouter le son que font les instruments de musique parce que si je fais ceci... (elle prend le triangle et tape une fois avec la baguette) cela me donne des idées qui sont ne sont pas les mêmes que si j'entends ça... (elle souffle dans la paille qui se trouve dans la bouteille d'eau).

E. : Ou si j'entends ça (elle tape plusieurs fois le tambourin avec les mains), ça ne donne pas les mêmes idées que si j'entends ça (elle fait sonner une petite cloche). Donc, vous inventez votre histoire en pensant aux instruments de musique que vous avez.

L'enseignante appelle le premier groupe à la table centrale pour qu'ils choisissent un instrument. Les enfants essayent les percussions et l'enseignante les guide dans ses essais. Quand elle appelle le deuxième groupe, les enfants du premier groupe sont encore là et tous les groupes se trouvent autour de la table en train de jouer les percussions. Les enfants changent plusieurs fois d'instrument. Certains enfants s'approprient un instrument particulier. Il y aussi des moments de négociation. On voit par exemple Raphaël en train d'échanger une cloche avec le tambourin d'Alexandra. D'autres enfants encore essayent différentes façons de produire des sons avec le même instrument.

\section{Créer des histoires collectivement}

Les groupes retournent à leurs tables. La phase de création des histoires commence. Les enfants discutent entre eux et produisent des sons avec des bouteilles et des percussions. L'enseignant passe entre les tables et suit les progrès de chaque groupe. Elle intervient en posant des questions et en donnant des conseils.

Extrait 12 :

Shanaël et Gary sont assis l'un devant l'autre. Shanaël a un tambourin dans la main, Gary fait de temps en temps des bulles avec la bouteille.

Shanaël : «eh tu vois ? eh bah...je dis (elle tape le tambourin avec la main), ou tu dis: «il va boire un cheval » allez... il était une fois un cheval qui buvait » hop !»

Gary : «eh bah, mais tous les chevaux boivent»

Shanaël : «oui, mais d'accord, est-ce que tu as une autre idée ? On pourrait (elle tape plus 
fort son tambourin), ou bien un gros géant (elle tape encore plus fort) ».

Les enfants sont en difficulté et elle propose à Shanaël de donner un exemple de début d'histoire.

E : vas-y, remontre Shanaël... il était une fois un cheval...

(Shanaël écoute) elle fait le bruit du cheval, elle secoue rythmiquement sa bouteilleinstrument.

Beaucoup d'enfants sont engagés dans la découverte de nouvelles sonorités. Sarah renverse la position de la baguette pour taper la cymbale. Shanaël tape avec le bâton du triangle sur un autre instrument. Alexandra et Marie produisent des sons avec les bouteilles, le tambourin er les cloches.

Alexandra secoue les cloches : ça c'est les cloches de Pâques.

Extrait 13 :

L'enseignante s'approche du groupe de Sarah, Eva et Caroline.

E. : «choisissez un son qui sera le bruit de la sorcière »

Eva prend une cloche

E. : «vas-y!» Eva secoue la cloche

E. : Il était une fois...

Sarah : Il était une fois une sorcière

E. : alors puisque c'est une sorcière, alors tu bouges ta cloche! Simultanément, Eva agite la cloche en produisant de petits sons saccadés.

E. : oui et alors qu'est-ce qui se passe avec la sorcière?

Sarah : qui faisait un bouillon (elle fait tourner sa bouteille en dessinant des cercles dans l'air)

E. : et alors qu'est-ce qui lui arrive?

Sarah : elle goûte le bouillon (elle tape son poignet contre la surface du tambourin).

Extrait 14 :

L'enseignante s'approche d'Alexandra.

E. : alors donc elle est dans la forêt, elle se promène

Alexandra : ça c'est le bruit de la forêt (elle joue du tambourin), non ça c'est la petite fille

E. : oui

Alexandra : ça c'est les cloches de Pâques (elle secoue les cloches)

E. : et quand est-ce qu'elles arrivent les cloches de Pâques dans ton histoire?

Alexandra (hésite) : c'est dans quel mois les cloches de Pâques?

E. : en avril bientôt

\section{Partager les histoires}

Les enfants en équipe restent assis à leur table, l'enseignante leur demande, équipe par équipe de raconter l'histoire aux autres.

Extrait 15:

$1^{\text {er }}$ groupe : Raphaël, Léo, Gilles et Maud

Raphaël : c'est l'histoire d'un cheval (Raphaël tape deux bâtons en bois l'un contre l'autre. Maud le suit au même rythme en secouant sa bouteille).

Raphaël : Léo tu dois faire (il indique la bouteille pleine d'eau)

Raphäl : et après il rencontre un poisson. (Léo fait des bulles en soufflant dans la paille).

Raphaël : et puis il décide de repartir. (ils font tous ensemble le bruit du cheval avec plusieurs instruments).

Raphaël : et puis il rencontre des soldats...

E. relance : il rencontre des soldats...

Raphaël : et après on avait dit que c'était terminé

$\mathrm{E}$ : ah d'accord, mais ce serait très chouette de savoir ce qui se passe après pour terminer l'histoire.

Léo : bah le cheval il devient un cheval soldat

E. : ah il devient... et qu'est-ce qu'il fait? il est à l'ordre des soldats? 
Raphaël : oui

E. : OK, faites-le (ils répètent le même bruitage).

$2^{\text {ème }}$ groupe, Caroline, Sarah, Eva

Sarah : il était une fois une sorcière (elle tape sur le tambour) qui était en train de préparer un bouillon (Caroline fait des bulles en soufflant dans la paille et la bouteille pleine d'eau). Et après elle boit le bouillon (Sarah frotte la main sur la surface du tambour) elle devient princesse (Sarah indique le tambourin à Caroline qui, comme Eva, commence à le secouer) et après elle boit (bulles de Caroline), et elle mange (les trois ensemble bougent les bouteilles en rythme), après elle redevient sorcière (Sarah frotte de nouveau la main sur le tambour) et elle va se promener (les trois secouent les bouteilles, Sarah et Caroline commencent à secouer en même temps leurs tambours).

$3^{\text {ème }}$ groupe, Alexandra et Marie

Alexandra: Il était une fois c'était le jour de Pâques, une petite fille dans la forêt (Alexandra

Joue le tambourin, Marie essaye de le prendre), tiens, embêtée, c'est à toi ça. Vas-y, c'est à toi. Et alors tout à coup (elle prend la bouteille er la secoue) il y a un cheval qui arrive au pas (elle secoue encore la bouteille de la même façon) et puis il va au galop (Marie secoue sa bouteille plus vite) et puis le jour de Pâques elle rentre à la maison et voit les aufs de Pâques (Alexandra bouge légèrement les cloches). Et voilà...

$4^{\text {ème }}$ groupe, Shanaël et Gary

Shanaël : les compagnons

Gary: il était une fois un cheval qui voulait avoir un compagnon (il fait des bulles) il parcourut le monde pour chercher son compagnon (il tape les noix de coco l'une contre l'autre) il trouva un chien, la pluie commença à tomber (Shanaël tape le bâton du triangle sur un autre instrument).

L'enseignante fait la synthèse.

E. : Très bien avec les instruments de musique vous avez réussi à faire des choses pas mal. Je crois que ce qui est aussi intéressant, c'est que dans votre histoire il y a vraiment un début comme vous faites : il était une fois, par exemple une petite fille qui se promenait dans la forêt, ou il était une fois une sorcière qui préparait son bouillon, ou il était une fois un cheval qui se promenait..., ou là il était une fois...

Shanaël : un cheval

E. : un cheval qui cherchait un compagnon et puis quelque chose qui se passe, il arrive... il parcourut le monde et rencontra un chien, ou ici elle rencontra les cloches de Pâques, là chez vous il rencontre un soldat, ici elle a mangé un bouillon et s'est transformée en princesse, d'accord. Et puis il faut quelque chose pour terminer votre histoire et ça...

Shanaël : chez nous c'est la chanson

E. : oui, mais peut être que juste avant de terminer par la petite chanson, c'est une très bonne idée, il faut une petite fin

Gary : oui on avait oublié de le dire... il rencontre beaucoup de monde et il va trouver encore plein d'autres compagnons, mais ceci c'est une autre histoire...

\section{3.- Retour au cycle de l'activité créatrice de l'imagination et à la dimension instrumentée de l'activité}

Nous pouvons faire deux premières remarques concernant cette situation pédagogique. D'une part, ce qui caractérise le laboratoire des rythmes par rapport à d'autres situations pédagogiques que nous avons observées est la présence constante d'expériences sensorielles. D'autre part, pour comprendre l'activité narrative, nous ne pouvons nous limiter à étudier le moment où les enfants s'assoient et commencent à créer l'histoire. La situation pédagogique doit être considérée comme un processus créatif complexe dans lequel plusieurs étapes intermédiaires contribuent à la réalisation du produit final.

Le modèle NAM (Decortis, 2008, Figure 5), issu d'un aller-retour entre nos observations de situations pédagogiques et le cycle de l'activité créatrice de l'imagination, rend compte de ce 
processus. La théorie instrumentale que nous mobilisons permet d'éclairer des aspects complémentaires.

Le modèle NAM intègre quatre phases: l'exploration (l'interaction de l'enfant avec son environnement), l'inspiration (la dissociation), la production (association et mutation) et le partage (raconter aux autres, présenter leurs histoires face à la classe). Il est important de remarquer que le déroulement du cycle n'est pas strictement linéaire et que les phases ne sont pas étanches. En effet, des boucles plus petites peuvent apparaître dans le cours de l'activité. On a observé par exemple une phase de partage au moment de la phase d'exploration ou de production, car les enfants écoutent les sons produits par les autres, ou de celle de l'inspiration. Des répétitions peuvent aussi se produire voire des creux.

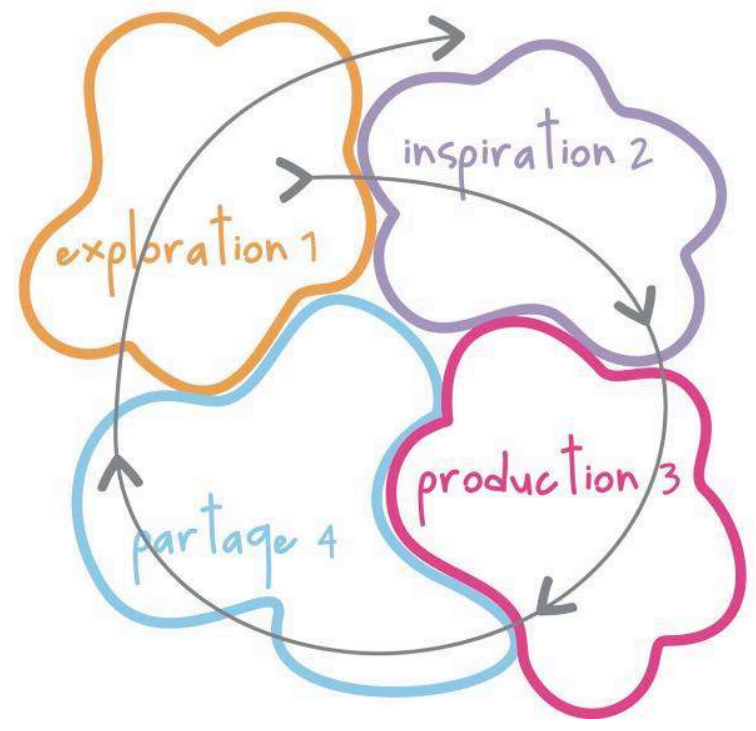

Figure 5 : Modèle d'Activité Narrative (NAM « Narrative Activity Model »)

Figure 5 : Narrative Activity Model (NAM)

La première phase du modèle concerne l'exploration de l'enfant de son environnement, qui peut être soit médiatisée par des instruments matériels diversifiés (livres, jumelles, marteaux, microscope), soit médiatisée par des relations sociales. Comme le montre Vygotski (1930/1983), l'imagination se construit à travers des éléments de la réalité qui sont rassemblés par l'expérience. Le cycle d'imagination créative se fonde sur la richesse et la variété de ces expériences. Durant cette phase, les impressions sont perçues comme un tout et seront par la suite dissociées. Cette dissociation implique que l'on donne de la pertinence à certains éléments et qu'on l'en écarte d'autres (Vygotski, 1930/1983).

Dans la phase d'inspiration, les impressions reçues durant la phase d'exploration du monde extérieur sont des touts complexes qui comprennent une grande quantité de parties différentes. Pour que les manifestations ultérieures de la fantaisie opèrent, la dissociation ou la division de ce tout complexe en ses parties est fondamentale comme le dit Vygotski (1930/1983). La dissociation implique aussi de donner une pertinence à certaines caractéristiques et pas à d'autres.

Durant la production, les éléments choisis préalablement vont être exploités pour produire un nouveau contenu à l'aide de diverses ressources. Cette phase se réfère notamment au mécanisme d'association qui est un moyen de réunir les éléments dissociés et transformés. En d'autres termes, durant la production, les objets qui auront été identifiés et choisis comme étant pertinents sont retravaillés pour produire de nouveaux contenus, et c'est aussi l'occasion d'explorer différents moyens d'expression et de découvrir ceux qui correspondent le mieux aux besoins et intentions expressives. De plus, la production offre une occasion 
d'externaliser le produit de son imagination et de ses émotions par diverses ressources (l'écrit, le dessin).

Enfin le cycle de l'activité ne sera complet que lorsque l'imagination aura pris corps, sera cristallisée en images externes (Vygotski, 1930/1983). Cette dernière phase du cycle ferme la boucle ouverte par la première phase : du monde réel vers l'imaginaire qui retourne ensuite vers le monde réel suffisamment partagé pour qu'il devienne objet d'expériences pour autrui, dernière phase concluante de l'imagination créatrice comme l'a décrit Vygotski (1930/1983).

Examinons maintenant ces quatre phases en lien avec le laboratoire des rythmes.

\section{1.- L'exploration}

Comme nous venons de le voir, lors de la première séance du « laboratoire des rythmes », les enfants confectionnent des objets : ils transforment des bouteilles de plastique en instruments de musique improvisés. Ils se dispersent dans le jardin à la recherche de matériaux (cailloux, bâtons en bois de différentes dimensions, fruits secs, etc). Les enfants sont libres dans l'exploration de leur environnement pour rechercher des éléments sonores, il s'agit d'un grand jardin.

L'objectif de l'enseignante dans la première partie de cette situation est d'inviter les enfants à décrire le contenu de leur instrument musical en vue d'explorer, de nommer et d'expliquer les différences entre sons. À ce stade il y a cependant un moment de partage, les enfants réunis en cercle, encouragés par l'enseignant, confrontent les sons produits par leurs instruments. En outre, l'usage d'un artefact quotidien (une bouteille en plastique) fait l'objet d'un détournement: sa transformation en un instrument musical. Ce détournement, bien connu des ergonomes sous le nom de catachrèse (Faverge, 1970 ; Rabardel, 1995), illustre bien une activité d'élaboration d'instruments par l'utilisateur. Ce détournement réalisé par les enfants, à l'invitation de l'enseignement, est opéré par le biais de l'attribution de nouvelles fonctions à l'artefact. En effet, sorti de son univers d'usage habituel, l'artefact acquiert de nouvelles propriétés par l'ajout de matériaux (cailloux, morceaux de bois, papiers, etc.).

En d'autres termes, les enfants contribuent à la conception de l'usage de simples artefacts pour en produire de plus complexes. Ils attribuent de nouvelles fonctions en fonction des caractéristiques des situations et des nouveaux buts qui leur sont assignés par l'enseignant: manipuler ces artefacts pour découvrir de nouveaux sons et explorer leurs propriétés sensorielles.

Le processus de détournement qui est guidé par l'enseignante se développe à deux niveaux : fonctionnel et sémantique.

\section{- À un niveau fonctionnel :}

La bouteille acquiert des propriétés sonores et se transforme d'un artefact qui contient habituellement du liquide en un «artefact pour produire des sons et des rythmes ». Au début de l'activité, pendant que l'enseignante donne des consignes, un enfant commence à produire des sons en pressant la bouteille. L'enseignante encourage cette exploration de sons et attire l'attention des autres enfants sur ce que fait un enfant (voir par exemple les Extraits 2 et 4). L'opération de remplissage des bouteilles complète cette transformation. Les enfants ont alors une série d'effets sonores que la bouteille vide ne permettait pas d'obtenir.

\section{- À un niveau sémantique :}

Les enfants apprennent progressivement à donner un nouveau signifié à la bouteille. Au fur et à mesure, sous l'impulsion de l'enseignante, ils prennent confiance avec le nouveau statut de l'artefact, ce qui les ouvre à de nouvelles explorations et expérimentations. En effet, une fois que l'artefact est reconnu comme instrument musical, les enfants commencent à explorer ses propriétés et à chercher de nouvelles façons de le manipuler. Cela est visible 
quand ils rentrent du jardin, ils sont debout autour de l'enseignante et chacun joue à son tour.

Les enfants expérimentent différentes façons de produire des sons avec les bouteilles, en les tapant, à l'invitation de l'enseignante, sur différentes parties du corps (tête, épaules, genoux), en changeant le rythme, en donnant des coups plus ou moins forts, etc.

Le changement de statut de l'artefact se poursuit quand les enfants expérimentent des sons produits en jouant à plusieurs. Les propriétés rythmiques de l'instrument sont ainsi explorées dans leurs dimensions combinatoires. À l'issue de ce moment d'exploration lorsque, sollicités par l'enseignant, les enfants commencent à jouer tous ensemble, l'un d'entre eux est tellement immergé dans cette nouvelle situation qu'il se met à danser.

La décoration des bouteilles contribue à la transformation sémantique de l'artefact, qui ne change pas sa fonction, mais comporte une redéfinition de ses propriétés esthétiques. L'enseignante met à la disposition des enfants des feuilles de papier coloré, colle et ciseaux et explique comment les utiliser pour la décoration (Extrait 7).

Une fois ce travail réalisé, l'artefact originel n'est alors plus reconnaissable. On peut aussi remarquer que le processus de transformation qui s'est mis en place lors de la première séance recommence lors de la deuxième séance. Les enfants qui avaient terminé la décoration de leur bouteille lors de la première séance continuent à inventer d'autres propriétés sonores. En effet, ils remplissent l'instrument avec de l'eau et, munis d'une paille, ils explorent des sons en soufflant dedans. Les sons obtenus seront souvent utilisés durant la phase de production narrative en raison des propriétés mimétiques de ces sons.

Ces mécanismes d'appropriation qui permettent l'élaboration de nouvelles ressources peuvent aussi être compris comme des processus de transformation qui sont typiques du jeu symbolique. Ils aident les enfants à se transporter en dehors de la réalité vers un contexte d'usage imaginaire (Harris, 2007).

Pour conclure autour de la phase d'exploration, ce qui caractérise le laboratoire des rythmes par rapport à d'autres situations pédagogiques est la présence constante d'expériences sensorielles (écouter, regarder). Dans d'autres situations que nous avons observées, l'exploration peut être initiée par au moins trois types d'activité :

— une activité sensorielle (écouter, regarder, sentir, toucher particulièrement travaillés lors d'une visite d'un musée, d'une promenade en forêt ou en classe) ;

- une activité descriptive, par exemple dans le «livre d'observations», les enfants décrivent une expérience vécue ;

- une activité manipulatoire, par exemple les enfants manipulent des éléments naturels (pâte à modeler ou ballons).

Ces deux dernières activités par lesquelles les enfants sont amenés à se représenter leur environnement peuvent être rapprochées des deuxième et premier niveaux que décrit Bruner (1996). Dans le mode iconique, l'information est représentée en termes d'images, le sujet construit des représentations à partir d'objets ou d'événements du monde réel. Les images saisissent la particularité des événements ou des objets, elles peuvent aussi fixer des repères auxquels le sujet peut confronter les événements afin de les classer. Dans le mode énactif (représentation énactive), l'information est représentée en termes d'actions spécifiées et habituelles.

\section{2.- L'inspiration}

Après cette première phase d'exploration des sons et de fabrication des instruments musicaux, l'enseignante dédie du temps à la réflexion et à l'analyse de l'expérience en demandant aux enfants de penser à ce qui est pertinent pour eux, de se souvenir d'éléments saillants qu'ils ont vécus et de les raconter oralement, graphiquement ou par écrit. L'enseignante incite les enfants à faire des choix, à retenir certains éléments et en écarter 
d'autres. Dans les termes vygotskiens, on peut penser que cela permet au processus de dissociation d'opérer.

Dans le laboratoire des rythmes, on peut noter l'importance d'esquisser les relations potentielles entre sons et idées. L'enseignante invite les enfants à discriminer les sons qu'ils produisent avec les instruments qui sont à leur disposition (Extrait 11).

Dans d'autres situations pédagogiques observées, les enfants sont invités à revisiter les impressions reçues, à se rappeler l'expérience vécue, ce qu'ils ont fait, s'ils ont aimé ce qu'ils ont fait ou vu, pourquoi ils ont aimé, ce qu'ils ont préféré. Mais il peut aussi être question de revenir sur une histoire entendue : revisiter l'intrigue, le début de l'histoire, ses parties, les personnages et leurs personnalités, les séquences préférées, ce que cette histoire leur dit ou leur enseigne. Il peut aussi être question de dissocier les propriétés de certains objets comme par exemple les sons d'un instrument.

Différentes modalités de dissociation ont été observées tels les jeux, l'échange et la discussion entre enfants et enseignant, le dessin ou l'écrit. Dans certaines situations, la dissociation peut être médiatisée et facilitée par des objets. Il peut être intéressant de revisiter l'expérience via des objets.

La dissociation peut se faire en cours d'activité ou post-hoc, après que l'exploration ait eu lieu. Dans le premier cas, les enfants expérimentent les propriétés de certains objets et sont amenés à distinguer celles-ci d'autres objets pour en définir leurs singularités. Par exemple, les enfants expérimentent les propriétés sonores d'instruments qu'ils ont fabriqués euxmêmes avec des bouteilles et divers matériaux trouvés dans le jardin de l'école et ils les comparent aux propriétés sonores des instruments de percussion qu'ils ont en classe. Dans le deuxième cas, les enfants reviennent sur une expérience passée et ils sont invités à se rappeler les événements vécus les plus signifiants pour eux et à partager l'expérience des autres.

Deux autres formes de dissociation consistent à revisiter des impressions premières ou des traces de ces impressions élaborées par soi. Dans le premier cas, les enfants revoient les impressions d'une expérience vécue qui n'a pas donné lieu à la constitution de traces et à la cristallisation de celles-ci sur un support. Dans le deuxième cas, les enfants retravaillent des traces qu'ils ont élaborées eux-mêmes précédemment, qui ont déjà été externalisées, comme par exemple des éléments qu'ils ont consignés préalablement sous la forme de descriptions ou de collages dans leur cahier d'observation.

La dissociation peut se faire spontanément par l'enfant ou être guidée par l'enseignant qui joue un rôle médiateur important. La dissociation au sens où l'entend Vygotski (1930/1983) est un processus individuel, chaque enfant définit pour lui-même les contours de son expérience. Mais ce travail peut aussi se faire avec l'appui du groupe, et dans ce cas tous les enfants peuvent entendre et éventuellement bénéficier de ce qui est important pour les autres. Les enfants peuvent également échanger leurs idées comme c'est le cas lorsque, tout en observant une scène, ils inventent une nouvelle histoire en relation avec celle-ci. C'est alors un mécanisme d'intersubjectivité qui se met en place comme Bruner (1996) le décrit et qui facilite la compréhension de ce que les autres enfants pensent ou ressentent. On pourrait même aller plus loin en disant que ces échanges servent de repères à chaque enfant pour comprendre la façon dont ses pairs opèrent une dissociation de l'expérience, à quoi leurs compagnons donnent de l'importance et ce qu'ils ne retiennent pas.

\section{3.- La production}

Durant la phase de production narrative du laboratoire des rythmes, les enfants font des associations entre les stimuli sensoriels provenant des instruments (musicaux et fabriqués) et leurs idées. L'objectif poursuivi par l'enseignant est de leur offrir une stratégie d'activation de l'imagination, en d'autres termes de créer des histoires «colorées» par le son. Ceci est 
visible quand l'enseignante dit aux enfants : «donc vous inventez votre histoire en pensant aux instruments de musique à votre disposition ».

Le rapport entre sons et idées s'inscrit dans un double mouvement (Figure 6).

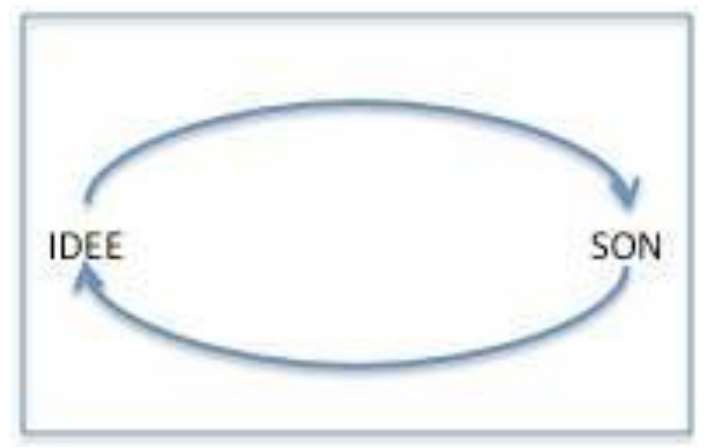

Figure 6 : Double mouvement du rapport entre sons et idées

Figure 6: Double movement of the relationship between sounds and ideas

\section{De l'idée au son :}

Le premier mouvement va des idées, telles qu'elles sont exprimées par des mots, aux sons. Par exemple, les enfants ont inventé une partie de l'histoire tels un personnage ou une phrase qui décrit un personnage (une sorcière dans l'extrait 13), et invités par l'enseignant, ils cherchent un son à associer à ce fragment narratif :

le son de la cloche est utilisé pour représenter le personnage qui entre en scène dans l'histoire. La médiation entre l'enseignant et les enfants vise à construire ces rapports entre idées (et mots) et sons.

\section{Du son à l'idée :}

Le deuxième mouvement entre éléments narratifs verbaux et sonores va dans une direction inverse : à partir d'un son, les enfants trouvent une idée pour l'histoire, c'est le son qui suggère l'idée. Ainsi dans l'extrait 12, l'idée d'un géant vient du changement de rythme. En percutant son instrument avec plus de force, Shanaël pense à un géant.

Dans ce douzième extrait, la médiation interpersonnelle s'inscrit dans les rapports intersubjectifs entre enfants. Dans l'extrait 14, la médiation interpersonnelle concerne les rapports intersubjectifs entre l'enseignant et un enfant. Les sons des cloches activent chez l'enfant une association avec les vacances de Pâques qui cherche donc une façon d'introduire cet élément dans son histoire. Les sons des cloches servent ainsi d'entrées sensorielles en tant qu'activateurs sémantiques.

Ainsi les formes de médiation interpersonnelles entre l'enseignant et les enfants sont subtiles et s'articulent autour de processus de génération des idées et des sons produits par les enfants qui manipulent les instruments. On observe ainsi un rapport du son à l'idée (et au mot), c'est le mouvement de l'idée au son et inversement du son à l'idée.

Dans la phase de production, on a observé dans d'autres situations pédagogiques l'importance de :

Inviter les enfants à identifier et réfléchir aux personnages d'une histoire. L'enseignante dit par exemple: "qui peut me dire quels sont les personnages les plus importants de l'histoire? ». Ensuite elle invite les enfants à penser et à définir leurs traits de personnalité.

Découper l'organisation structurelle de l'histoire. Le travail de dissociation encouragé par l'enseignant peut aussi porter sur l'organisation structurelle de l'histoire, sur la façon dont les événements sont organisés et sur la chaîne linéaire des événements. Les enseignants demandent par exemple aux enfants de choisir les séquences qu'ils ont préférées, de les 
écrire et de les illustrer.

En outre, nous avons observé que durant la production, les enfants écrivent, lisent ou dictent oralement des fragments d'histoires à l'adulte, jouent corporellement en exprimant leurs émotions et dessinent en donnant une forme iconique aux histoires racontées oralement. La dictée à l'adulte en première année primaire $(\mathrm{CP})$ est un moment particulier où l'enfant offre ses premières idées qu'il discute individuellement avec l'adulte. En effet, les enfants qui apprennent à écrire sont invités à dicter leur histoire à l'adulte et, ensuite, à recopier cette histoire dans un cahier. Deux instruments sont utilisés pour cette activité interactive entre l'adulte et l'enfant, le cahier de brouillon et le cahier d'histoire. Comme indiqué précédemment le cahier de brouillon est un outil partagé entre l'enseignant et l'enfant. L'enseignant écrit dans celui-ci l'histoire qui est dictée par l'enfant. Il est ensuite utilisé par l'enfant pour recopier l'histoire dans un cahier qui lui est personnel, le cahier d'histoire. Les seules marques que l'enfant apporte au cahier de brouillon sont celles de barrage de mots qui ont déjà été copiés. Tandis que le cahier d'histoires ne contient que des traces de l'enfant porteuses de son expression personnelle.

La production est marquée par la multiplicité des ressources utilisées. Le dessin, l'expression verbale ou la création de costumes sont autant d'outils que les enfants peuvent utiliser pour produire leurs histoires. Par exemple, les enfants sont invités à dessiner les personnages qu'ils ont choisis d'incarner avant de fabriquer eux-mêmes les costumes qu'ils porteront lors du partage de leurs histoires devant une assemblée.

La production peut être individuelle ou collective. Soit l'enfant dicte à l'adulte son histoire qui la copie dans un cahier de brouillon. Soit les enfants produisent collectivement l'histoire, chacun proposant une partie de celle-ci. Dans ce cas, les enfants négocient les parties communes qui doivent être écrites ou dessinées et ils décident ensemble des personnages, de leurs caractéristiques et des dialogues.

Les médiations interpersonnelles entre l'enseignant et l'enfant sont subtiles et complexes. Il peut s'agir pour l'enseignant de superviser l'organisation du contenu narratif, d'assurer que l'histoire soit conforme avec quelques règles de sa construction, et que le texte soit cohérent et riche. Mais sa médiation ne s'arrête pas là. En effet, plusieurs enseignants ont expliqué que lors de la dictée à l'adulte, ils visualisent l'histoire de l'enfant et que cette visualisation leur donne des repères pour orienter leurs questions qui traduiront leur intérêt et leur attention aux aspects les plus signifiants. Leurs objectifs étant d'élargir et d'enrichir la capacité de l'enfant à s'exprimer personnellement à travers son histoire. C'est la partie la plus importante du rôle moins visible de l'enseignant comme le montre Engel (1999), interagir avec l'enfant en répondant à son récit de façon appropriée et sensible. Il s'agit par exemple d'éliciter l'expression des sentiments, des émotions, des sensations et de l'imagination de l'enfant : E. : «c'était comment... c'était doux, c'était...», «Qu'est-ce que tu entendais? « Qu'est-ce que tu voyais? ».

Lorsque la production se fait avec un groupe d'enfants, l'enseignant vise à susciter la discussion entre enfants et à faire en sorte que chaque enfant contribue à l'histoire, mais aussi à les aider à négocier des contenus qui soient communs et qui soient cohérents. Dans cette phase de production, les enseignants considèrent que leur rôle est multiple :

- fournir aux enfants des moyens qui leur permettront de réaliser leurs intentions, par exemple proposer des activités narratives à travers lesquelles ils pourront exprimer leurs idées, leurs émotions et leurs vécus,

— construire des repères et les aider à faire des choix en leur présentant plusieurs types d'histoires et de structures ;

— offrir des techniques et du matériel à partir desquels les enfants pourront travailler afin d'enrichir leur potentiel ;

— encourager l'enfant "à aller plus loin..."; en lui posant des questions du type : "Et qu'est- 
ce qui se passe après ?", "As-tu envie de dire autre chose? ";

- aider l'enfant à court d'idées et stimuler son envie de raconter quelque chose, en posant des questions, comme par exemple: "Y a-t-il quelque chose que tu aurais envie de raconter?".

\section{4.- Le partage}

Ainsi que nous l'avons évoqué, le cycle de l'activité ne sera complet que lorsque l'imagination aura pris corps, sera cristallisée en images externes (Vygotski, 1930/1983). Durant la phase de production, les enfants externalisent déjà le produit de leur imagination par la dictée à l'adulte. Au moment du partage, les enfants racontent leurs histoires aux autres enfants, présentent leurs jeux théâtraux devant leurs parents, expliquent leurs dessins et leurs histoires face à la classe et l'enseignant. Toutes manifestations externalisées qui influencent le monde extérieur de l'enfant. La phase de partage ajoute une dimension, car les productions externalisées commencent à exister dans l'environnement de l'enfant et peuvent avoir un effet sur les autres (enfants, parents, enseignants).

Cette dernière phase du cycle ferme la boucle ouverte par la première phase : du monde réel vers l'imaginaire qui retourne ensuite vers le monde réel suffisamment partagé pour qu'il devienne objet d'expériences pour autrui, dernière phase concluante de l'imagination créatrice comme l'a décrit Vygotski (1930/1983).

Plusieurs modalités d'expressions: verbale, corporelle, musicale peuvent caractériser le partage. Les enfants parlent de leurs expériences, ce qu'ils ont fait, pourquoi ils aiment telle chose, «que ferait-il si...». En partageant leurs histoires, les enfants s'expriment à travers leur corps en utilisant l'espace, par la danse par exemple, ou par l'utilisation de costumes. Les enfants peuvent utiliser l'une de ces modalités ou plusieurs à la fois.

Dans le laboratoire des rythmes, les enfants alternent entre histoire orale et musicale comme dans l'extrait 15 ( $3^{\text {ème }}$ groupe).

Le partage peut être médiatisé par un instrument diégétique, l'instrument se trouve à l'intérieur de l'espace de l'histoire (exemple l'instrument de musique), et il peut servir à rythmer l'histoire, ou extradiégétique, l'instrument se trouve à l'extérieur de l'espace de l'histoire et sert de support au partage, par exemple lorsque l'enfant s'aide d'un cahier comme aide mémoire et/ou pour montrer son histoire aux autres.

Le partage peut se faire dans des espaces dédiés et collectifs (par exemple le gymnase de l'école), soit dans des espaces habituels, en classe.

Le partage peut avoir été préparé et contribuer à créer des traces qui ont une certaine permanence (le temps de l'année scolaire): un poster, un tableau, ou au contraire être éphémère et ne durer que le temps de la situation pédagogique.

Le partage peut être l'occasion d'établir un échange avec ceux qui viennent assister à la production narrative et qui n'ont pas participé à la création (spectateurs, parents).

La médiation interpersonnelle entre l'enseignant et les enfants est fondamentale durant la phase de partage. L'enseignant invite les enfants à exprimer ce qu'ils ont fait et les raisons qui ont poussé chacun à produire leur histoire. Un exemple issu d'une observation d'une situation pédagogique durant laquelle chaque enfant est invité à raconter une histoire autour de la rencontre de ses parents le montre. L'enseignante montre à tous les enfants les histoires de chacun. La discussion est orientée par une prise de conscience qu'autour d'une même thématique, chaque enfant donne une signification personnelle et différente à un événement de sa vie, et qu'il est important de reconnaître le point de vue de chacun. L'extrait suivant permet d'illustrer ce point :

Enseignant : "Lorsque vous entendez toutes ces histoires, qu'avez-vous envie de dire?". Enfant : "Les dessins sont beaux et c'est bien écrit. Et c'est beau de voir tous les dessins et 
les histoires ».

Enseignant : "Pourquoi est-ce beau d'entendre les histoires des autres?».

Enfant : "Sur un même thème, tous les enfants ont raconté une histoire différente ».

Enseignant : "Oui sur un même sujet, chacun de vous a raconté une histoire différente et c'est pourquoi c'est intéressant. Et toutes les histoires sont belles ainsi que tous les dessins».

\section{4.- Conclusions}

Les instruments mobilisés par l'enseignant sont diversifiés, instruments matériels et psychologiques. Ces derniers tournés vers le développement de l'activité de l'enfant permettent de «créer des aptitudes nouvelles » (Leontiev, 1976). Sous le guidage de l'adulte, l'enfant acquiert les éléments culturels mis en patrimoine dans l'outil. Les instruments sont tout à la fois des bouteilles, des objets glanés dans le jardin, des instruments musicaux, des pailles, etc. Ils associent tout à la fois la production de sons, les dessins, les mimes. Guidé par l'enseignant, l'enfant s'approprie ces instruments pour constituer des ressources de sa propre activité et s'ouvrir à ses propres possibilités créatives.

Les médiations interpersonnelles entre l'enseignant et l'enfant peuvent être articulées au cycle de l'activité créatrice de l'imagination. En effet, le cycle peut offrir à l'adulte un cadre pour guider l'enfant dans l'appropriation de l'instrument et dans la construction de nouvelles ressources internes.

Pour contribuer au développement de ressources créatives du sujet, les enseignants opèrent à deux niveaux: d'une part, accompagner les détournements des artefacts, d'autre part développer un « laboratoire intérieur ».

\section{1.- Accompagner les détournements des artefacts à un niveau fonctionnel et sémantique}

Les enseignants incitent les enfants à tourner leur attention vers l'expérience singulière et vers l'outil. Les enseignants aident les enfants à réaliser des catachrèses, c'est-à-dire à opérer des détournements des artefacts à un niveau fonctionnel et sémantique. Ainsi, les enseignants guident les enfants à opérer des genèses instrumentales à la fois dirigées vers eux-mêmes (instrumentation) et dirigées vers l'artefact (instrumentalisation). Il y a à ce stade tout à la fois un travail sur l'ouverture des ressources intérieures et sur les variations que le sujet peut entretenir avec son environnement.

Plus généralement, nos observations à l'école de détournement de l'artefact pointent les maintes occasions que saisissent les enfants de transformer les propriétés et le signifié des artefacts. Dans leurs mains un bâton devient une épée ou une baguette magique, une ombre devient un personnage, une feuille de papier un billet de banque. Rodari (1997) a bien montré qu'en transportant dans leur monde des objets abandonnés par leurs parents ou par des adultes, les enfants leur donnent une nouvelle vie, un réveil cassé devient un jouet, une bouteille en plastique devient un instrument de musique.

L'enseignant s'appuie sur cette ressource naturelle des enfants pour leur proposer une transformation de l'artefact. L'attribution de nouvelles fonctions à l'artefact (la bouteille en plastique), encouragée par l'enseignant, est liée aux caractéristiques de la situation, aux matériaux disponibles (bâtons, cailloux, pailles) et aux objectifs assignés par l'enseignant. Le détournement opéré est donc bien un indice du fait que les utilisateurs contribuent à la conception des usages de l'artefact (Rabardel, 1995). C'est bien ce que les enfants apprennent ici, c'est-à-dire élaborer des instruments destinés à être insérés dans leur activité en fonction de leurs objectifs. Ils apprennent ainsi à changer la fonction de l'artefact qui n'apparaît plus comme une propriété fixe et intangible, mais qui peut résulter d'un processus de transformation. 
En outre, nous pouvons observer que ces changements d'attribution portent sur la signification de l'usage de l'artefact. En prenant confiance avec le changement de statut de l'artefact, les enfants apprennent à lui donner un nouveau signifié (il ne s'agit plus d'une banale bouteille en plastique, mais bien d'un instrument musical), ils apprennent à le manipuler de diverses façons (le taper sur le corps), mais ils apprennent aussi à redéfinir ses propriétés esthétiques (décorer la bouteille ne change pas sa fonction, mais opère une transformation sémantique).

Cette observation appelle trois grandes lignes de réflexions et perspectives d'études à venir, l'une en lien avec les concepts de jeu chez Vygotsky (1978), la deuxième en lien avec le concept de genèse instrumentale chez Rabardel (1995), enfin la troisième en lien avec le concept de bricoleurs bien décrits par Ackermann (2011).

1. Le jeu est un facteur de développement de l'enfant très important comme l'a souligné Vygotski (1978). Par le jeu l'enfant crée une situation imaginaire, une petite fille imagine être une maman et la poupée être son enfant, ce qui amène l'enfant à acquérir des règles d'action qui peuvent rester inaperçues dans la vie réelle, mais qui sont exprimées par l'enfant durant le jeu, voire explicitées, discutées, négociées entre enfants au cours du jeu. Le rôle que l'enfant accomplit et sa relation à l'objet (si l'objet a changé sa signification) proviendront toujours des règles qu'il exprime (Vygotki, 1978). Ainsi, dans notre situation pédagogique, le dispositif mis en place par l'enseignant qui consiste à construire des instruments musicaux à partir de bouteilles, et les règles d'action qui sont développées, crée une situation imaginaire assortie de diverses possibilités d'action. Vygotski montre que dans le jeu, les objets perdent leur force déterminante (chez le jeune enfant, les objets dictent la conduite à avoir par exemple une porte demande à être ouverte et fermée, ils ont une force motivante inhérente), l'enfant peut voir un objet, mais agir différemment en relation avec ce qu'il voit. C'est à partir de là, selon Vygotski, que l'enfant apprend à agir en mobilisant ses ressources cognitives, à mobiliser ses aspirations et souhaits, plutôt que de répondre à des contraintes situationnelles. Ainsi l'action dans une situation imaginaire apprend à l'enfant à guider sa conduite pas seulement en regard de la perception immédiate des objets ou des situations immédiates, mais aussi par la signification de cette situation (Vygotski, 1978, p. 97). L'action peut commencer à être déterminée par des idées plutôt que par des objets en tant que tels, par exemple un manche à balai peut devenir un cheval. Et ceci constitue un renversement de la relation de l'enfant au réel et aux situations concrètes. Or l'enfant n'acquiert pas cela en une fois, car il est difficile pour l'enfant de découpler la pensée (la signification d'un mot) de l'objet. Dans le jeu, l'enfant crée la relation entre le sens et l'objet dans lequel la nature sémantique domine et détermine le comportement. La signification est libérée de l'objet avec lequel il a été directement fusionné auparavant. Dans le jeu, l'enfant se concentre sur la signification découplée par rapport à l'objet, mais qui n'est pas découplée dans l'action réelle avec des objets réels. En conclusion, nous pouvons dire que le jeu libre chez l'enfant est trop souvent relégué comme secondaire par rapport aux apprentissages jugés fondamentaux, or le rôle du jeu dans le développement et ses liens avec l'activité narrative et créative méritent davantage d'attention et l'ergonomie peut y contribuer.

2. Dans la perspective de la théorie instrumentale, nous observons dans la situation que nous venons de présenter, une genèse instrumentale guidée par l'adulte en direction des enfants. Les élaborations instrumentales des enfants qui sont dirigées vers l'artefact relèvent de la dimension instrumentalisation: institutions de nouvelles fonctions, attribution de propriétés, transformation de l'artefact dans sa structure et son fonctionnement. Nous avons examiné cela en détail. Les élaborations instrumentales des enfants qui sont dirigées vers eux-mêmes relèvent de la dimension instrumentation. C'est que nous allons examiner dans le paragraphe 4.2. D'autres études sont nécessaires pour aller plus loin dans cette voie en particulier pour bien comprendre le statut des schèmes d'utilisation, ce que nous n'avons pas fait dans cette étude, et pour spécifier les différents 
types de schèmes qui composent la classe des schèmes d'utilisation.

3. La façon de faire de l'enseignante et celle des enfants, renvoient à ce qu'Ackermann (2011) appelle une culture «de bricoleurs, fabricants, pirates informatiques, amateurs » qui engendre de nouveaux rapports aux choses. Comme elle le souligne, un bricoleur est un « touche-à-tout » qui sait comment se débrouiller avec ce qui est à portée de la main, il est l'expert en diverses tâches qui assemble des choses préexistantes de nouvelles façons. Plus que la génération précédente, les enfants d'aujourd'hui désirent rassembler, prendre, créer et négocier des objets. Comme les auteurs d'aujourd'hui, les bricoleurs aiment tordre les choses qu'ils trouvent, leur donner une deuxième vie. Cela laisse supposer que les générations prochaines perfectionneront leurs compétences techniques, inventeront de nouvelles façons de faire (traitement, fabrication, invention), développeront de nouvelles ressources et confiance dans leurs capacités de réparer, affiner, transformer, itérer. Comme Ackermann le souligne, si les possibilités et les ressources sont données aux jeunes, plutôt que de consommer, ils créeront et recycleront. C'est, nous semble-t-il, une voie d'étude innovante pour l'ergonomie pour les prochaines années.

\section{2.- Développer un « laboratoire intérieur » : médiations interpersonnelles réflexives}

Comme nous l'avons souligné, l'enseignante incite les enfants à diriger leur attention vers eux-mêmes. Tout se passe comme si, à l'aide de multiples ressources qui associent la production de sons et le langage, elle engage les enfants à constituer une sorte de «laboratoire intérieur». En effet, des outils du réel sont expérimentés par les enfants qui sont invités à les écouter, à porter attention à leurs variations, à écouter leurs échos en eux, dans leur voix intérieure. À l'aide de la bouteille, transformée dans une direction fonctionnelle et sémantique, les enfants peuvent produire des sons et s'en inspirer pour produire leurs récits. Il s'agit d'instruments transitionnels au sens où Bationo-Tillon, Folcher et Rabardel (2010) l'entendent. En effet, la bouteille est un «artefact inanimé auquel le narrateur confère un certain nombre de propriétés », elle est fabriquée par l'enfant qui lui donne un nouveau statut, non pas d'aide mémoire, de témoin, mais bien davantage de «médiateur à idées». L'enseignante invite les enfants, tout à la fois, à faire des discriminations fines entre sons et à écouter les échos qu'ils produisent en eux. Mais elle les incite aussi à construire des liens entre le réel et l'imaginaire et, par le récit, à réélaborer de façon créative leurs impressions. Ces façons de faire de l'enseignante ouvrent les enfants à leur propre créativité.

Nous considérons qu'il s'agit là de médiations interpersonnelles réflexives, car l'enfant est invité par l'enseignante à apprendre à se connaître, à se transformer. Mais nous considérons qu'il s'agit de médiations interpersonnelles réflexives spécifiques. Elles consistent à ouvrir la palette des ressources du sujet, à élargir ses possibilités de dissociations et d'associations, et à générer des matériaux qui seront utiles pour la production. Un son produit par l'enfant, renvoie celui-ci à écouter ce qu'il imprime en lui, à choisir parmi ces impressions celle qui lui convient le mieux, et ensuite, par association, à produire des idées et des mots. En d'autres termes, le langage intérieur devient un « laboratoire intérieur» dans lequel l'enfant fait des essais, écoute les échos en lui et utilise ce matériau pour produire des idées.

Ce processus renvoie bien aux mécanismes de l'imagination dont nous avons parlé plus tôt et qui constitue un point d'appui pour le développement de la créativité. Ce que l'enfant voit ou entend est suivi d'un processus de réélaboration du matériel qui comprend la dissociation et l'association.

Il est évident que ces médiations interpersonnelles réflexives sont différentes des médiations collaboratives qui se déploient davantage sur le volet de la collaboration (atteindre des buts communs au sein d'activités collectives). Nous considérons que ces médiations entraînent et renforcent la capacité des enfants à imaginer des possibilités diverses et alternatives. Il s'agit 
d'une capacité évidente dans le jeu symbolique qui émerge très tôt dans le développement de l'enfant comme Harris (2007) le souligne et qui ne disparaît pas nécessairement, mais peut au contraire durer une vie entière. En d'autres termes, nous pensons que ces médiations interpersonnelles réflexives ont une fonction de développement de la créativité des enfants.

Ainsi dans la perspective d'une théorie instrumentale étendue (Rabardel, 1999), notre étude permet d'éclairer les dimensions de l'activité qui contribuent au développement de la créativité des sujets. En effet, ce que nous pointons ici est que les enfants produisent des fonctions instrumentales orientées vers la découverte de leurs imaginations.

\section{3.- Élargissement du récit à l'activité narrative}

L'élargissement du concept de récit à l'activité narrative est au cœur de nos recherches. Une unité d'analyse, plus ample que le récit, adoptée par des chercheurs en narratologie et développement tels que Bamberg (1997), MacCabe (1997) ou Engel (1999), doit, de notre point de vue, être envisagée. L'activité narrative nous semble plus appropriée, car elle permet de rendre compte de la production narrative en tant qu'activité multi-instrumentée et créative, à donner une place centrale à ce que fait le sujet en situation quand il construit une histoire, à ce qu'il fait dans un empan temporel large, ainsi qu'aux médiations instrumentales qui opèrent lors de la construction d'un récit, et les ressources que le sujet construit. De notre point de vue, la mise en relation de l'activité narrative et du cycle de l'activité créatrice de l'imagination (Vygotski, 1930/1983) permet de comprendre la façon selon laquelle l'enfant assemble les informations de l'expérience pour construire un récit qui répond à ses curiosités et ses besoins. Puisant dans les matériaux pris dans la réalité, l'enfant nourrit son imagination. La médiation interpersonnelle qui opère grâce aux échanges que l'enfant entretient avec ses pairs et d'autres sujets enrichit les représentations que l'enfant se construit. Ainsi réalité et imagination ne sont pas antithétiques, mais bien assemblées de façon productive et progressive via le récit.

Le modèle NAM décrit la façon dont le sujet fait l'expérience du monde extérieur, élabore des impressions reçues, les assemble d'une façon nouvelle et les partage avec d'autres. Ce qui est un processus psychologique continu, et qui se déploie comme un tout dans l'activité de l'enfant, peut être décomposé non pas dans l'esprit des enfants, mais dans la structure d'une situation pédagogique organisée par l'enseignant. Les activités narratives à l'école incluent une centration sur chacune de ces phases, souvent en séquences linéaires, parfois avec des répétitions, parfois en creux. C'est la façon dont les différentes phases chronologiques et structurelles de l'activité narrative peuvent se saisir du cycle de l'imagination créative.

L'activité narrative est très souvent discontinue comme le montrent Bationo-Tillon (2006), Bationo-Tillon et al. (2010). Elle se construit par ajouts successifs sur des périodes qui peuvent être longues comme pour les voyageurs explorateurs. Rendre compte du caractère diachronique de l'activité narrative est ainsi un enjeu de compréhension de la volatilité de l'activité narrative dans sa discontinuité. Si pour l'adulte c'est souvent le langage qui permet de relier les contenus d'expériences diverses et discontinues, pour les enfants c'est aussi la collection de petits objets (gri gri, morceaux de papiers, cailloux), sur le lieu de leur expérience qu'ils transporteront avec eux à la maison, à l'école pour constituer une collection imaginaire. Ces objets collectés durant l'exploration serviront de support durant la phase d'inspiration pour créer un univers narratif qui n'est pas uniquement nourri par des histoires écrites bien formées. Nous donnons souvent trop d'importance aux dimensions écrites et linguistiques des histoires comme le souligne Ackermann (2001). Il est intéressant d'envisager le développement des compétences narratives dans leur ensemble en considérant les multiples moyens d'expression dont les enfants sont friands.

Comprendre l'activité narrative dans ses divers empans temporels est une nécessité évidente. Le cycle de l'activité créatrice de l'imagination que décrit Vygotski, en s'inscrivant dans le 
long terme, ouvre des pistes d'investigation intéressantes qui ne sont pas explorées par la psychologie de la créativité notamment (Lubart, 2005). Il peut nous permettre de rendre compte des dimensions productives et constructives de l'activité (Rabardel, 2005) qui s'élaborent dans le long terme, et qui peuvent être discontinues, mais aussi dans le moyen ou le court terme.

Nous pensons que les perspectives que nos recherches ouvrent sont nombreuses sur un plan empirique, pragmatique et épistémique. Premièrement le modèle est un outil d'analyse des situations de travail, culturelles ou pédagogiques dans lesquelles la production de récits est significative. Nous avons peu exploré la troisième forme de relation entre imagination et réalité qui est émotionnelle. De futures recherches afin de mieux comprendre la prise en compte des affects présents et actifs dans de nombreuses situations (Cahour, 2010) et l'influence des émotions sur l'imagination seraient très intéressantes.

Deuxièmement le modèle peut outiller la réflexion pour envisager des pistes de transformation et de conception de situations de médiations culturelles et des dispositifs techniques qui soient adaptés. Le modèle est en effet un outil pour penser les technologies mises à disposition des enfants tant dans leur conception que leur évaluation. Il a déjà été un outil précieux pour concevoir des environnements pour les enfants qui soutiennent leur désir de s'engager activement dans la production de récits en particulier pour concevoir des technologies tangibles (Decortis et al. 2001). Par ailleurs, nous étudions la façon dont on peut repenser les outils de médiation culturelle et concevoir des technologies qui n'entravent pas l'activité narrative (Decortis \& Bationo-Tillon, sous presse). Dans cette voie le modèle aide à comparer différentes situations dans lesquelles le récit s'avère pertinent, en particulier pour fournir des recommandations aux médiations culturelles (Bationo-Tillon \& Decortis, 2012).

\section{Remerciements}

Mes remerciements vont en premier lieu aux enfants qui nous ont ouvert quelques petites fenêtres de leur imaginaire et aux enseignants de l'école Hamaïde à Bruxelles, en particulier, Béatrice Gizzi, Martine Balencour, Isabelle Goldschmidt, Nathalie Eloy, Véronique Rahier. Mes remerciements vont ensuite à mes collègues de Paris 8 pour nos échanges autour de cet article, Anne Bationo-Tillon, Pierre Rabardel, Gérard Vergnaud, à mes étudiantes Laura Polazzi et Berthe Saudelli qui ont largement contribué aux observations à l'école, et enfin à Édith Ackermann, Françoise Détienne, Valery Nosulenko et aux experts anonymes de la revue Activités pour leur lecture attentive et la qualité de leurs remarques constructives.

\section{BIBLIOGRAPHIE}

Ackermann E. (2001). Bambini Digitali, Strumenti narrativi, scrittura dialogica. Tecnologie Didattiche, 24(3), 2001, 24-48.

Ackermann, E. (2011). Minds in Motion, Media in Transition. Growing up in the digital age: Areas of change. In Children are our future. Child Research Net. Japan. URL : http://www.childresearch.net/RESOURCE/RESEARCH/2011/ACKERMANN.HTML

Ananny, M. \& Cassell J. (2001). Telling Tales: A new toy for encouraging written literacy through oral storytelling. Paper presented at the Conference of the Society for Research on Child Development, Minneapolis, MN.

Bamberg, M. (1997). Narrative development: six approaches. Londres: Lawrence Erlbaum.

Bationo-Tillon, A. (2006) Pratiques des activités narratives instrumentées : Une analyse diachronique et structuro-fonctionnelle en amont de la conception. Doctorat d'ergonomie, SaintDenis: Université de Paris 8.

Bationo-Tillon, A., \& Decortis, F. (2011). L'activité narrative dans un contexte muséal - étude de cas : l'exposition Monumenta consacrée à Anish Kapoor. In V. Folcher et al., Arts et Handicap. Médiations humaines et techniques pour un accès durable à l'offre culturelle. Rapport de recherche rendu dans le cadre du financement P.A.R.I 2010-2012 \& en partenariat Université Paris 8 - CNAP - Association Perspectives et Actions. 
Bationo-Tillon, A., Folcher V., \& Rabardel, P. (2010) Les instruments transitionnels : une proposition pour étudier la diachronie des activités narratives. Activites. 7(2), 63-83.

Beaujouan, J. (2011). Contributions des récits professionnels à l'apprentissage d'un métier. Le cas d'une formation d'ergonomes. Thèse de doctorat d'ergonomie, Bordeaux : Université de Bordeaux Segalen.

Beaujouan, J. \& Daniellou, F. (2012). Les récits professionnels dans une formation d'ergonomes. Le Travail Humain, 75(4), 353-376.

Bruner, J.S. (1996). L'éducation entrée dans la culture. Paris: Retz.

Cahour, B. (2010). Émotions, affects et confort comme nouveaux déterminants de l'activité et de l'usage. In G.Valléry, M. Zouinar, \& M. C. Le Port (Eds.), Ergonomie des produits et des services médiatisés : nouveaux territoires, nouveauxenjeux (pp. ). Paris: PUF.

Cassell, J., \& Ryokai, K. (2001). Making space for voice: Technologies to support children's fantasy and storytelling. Personal Technologies, 5(3), 203-224.

Decortis, F. (2008). L'activité narrative et les nouvelles technologies pour les enfants. Habilitation à Diriger des Recherches. Université Paris 8.

Decortis, F., \& Bationo-Tillon, A. (sous presse). "Once upon a time, there was a fairy who walked in paradise": the child finalised, mediatised and creative narrative activity. International Journal of Arts and Technology.

Decortis, F., \& Rizzo, A. (2002). New active tools for supporting narrative structures. Personal \& Ubiquitous Computing, 6, 416-429.

Decortis, F., Rizzo, A., Daele, L., Polazzi, L., \& Saudelli, B. (2001). Nouveaux instruments actifs et activités narratives. Pogo : vers un espace de création située. Revue d'Interactions HommesMachines, 2(2), 1-30.

Decortis, F., Rizzo, A., \& Saudelli, B. (2003). Mediating effects of active and distributed instruments on narrative activities. Interacting with Computers. 15(6), 801-830.

Dewey, J. (1987). L'art comme expérience. Paris: Folio, essais.

Engel (1999). The stories children tell. Making senses of the narratives of childhood. New York: Freeman and Company.

Faverge, J.M. (1970). L'homme agent d'infiabilité et de fiabilité du processus industriel. Ergonomics, 13(3), 301-327.

Folcher, V., \& Rabardel, P. (2004). Hommes, artefacts, activités : perspective instrumentale. In P. Falzon (Ed.). Ergonomie (pp. 251-268). Paris: PUF.

Guha M. L., Druin, A., Montemayor, J., Chipman, G., \& Farber, A. (2007). A Theoretical Model of Children's Storytelling using Physically-Oriented Technologies (SPOT). Journal of Educational Multimedia and Hypermedia, 16(4), 389-410.

Harris, P.L. (2007). L'imagination chez l'enfant. Son rôle crucial dans le développement cognitif et affectif. Paris: Retz.

Lubart, T. (2005). Psychologie de la créativité. Paris: Armand Colin.

MacCabe, A. (1997). Developmental and cross-cultural aspects of children's narration. In M. Bamberg, Narrative development : six approaches (pp. 137-174). Londres: Lawrence Erlbaum.

Malaguzzi, L. (1987). I cento linguaggi dei bambini: Narrativa del possibile. Proposte di bambini delle scuole dell'infanzia di Reggio Emilia. Italie.

Marchand, A.-L. (2011). Les retours d'expériences dans la gestion de situations critiques. Activités, 8(2), 100-113. http://www.activites.org

Marchand, A.L., \& Falzon, P. (2011). Usages pédagogiques de la pratique anecdotale dans la formation à la gestion des risques. Psychologie du travail et des organisations.

Rabardel, P. (1995). Les hommes et les technologies. Paris: Armand Colin.

Rabardel, P. (1999). Le langage comme instrument ? Éléments pour une théorie instrumentale étendue. In Y. Clot (Ed.), Avec Vygotski (pp. 241-266). Paris: La Dispute.

Rabardel, P. (2005). Instrument subjectif et développement du pouvoir d'agir. In P. Rabardel \& P. Pastré, P. (2005). Modèles du sujet pour la conception. Dialectiques activités développement (pp. 11-29). Toulouse: Octarès. 
Rabardel, P., \& Béguin, P. (2005). Instrument mediated activity: from subject development to anthropocentric design. Theoretical Issues in Ergonomics Science. Vol. 6, No. 5, September 2005, pp. 429-461.

Raffle, H., Vaucelle, C., Wang, R., \& Ishii, H. (2007). Jabberstamp: embedding sound and voice in traditional drawings. Proceedings of the Interaction Design for Children Conference, Danemark, pp. 137-144.

Rodari, G. (1991). Grammaire de l'imagination. Introduction à l'art d'inventer des histoires. (Trad. française. De R. Salomon). Paris: Rue du Monde.

Ryokai, R. \& Cassell, J. (1999). StoryMat: a play space for collaborative storytelling. Proceedings of the Conference on Human Factors in Computing Systems, USA, pp. 272-273.

Vaucelle, C. (2010). Play it by eye, frame it by hand! Gesture Object Interfaces to enable a world of multiple projections? PHD Massachussets Institute of Technology.

Vergnaud G. (2002) Forme opératoire et forme prédicative de la connaissance. Conférence introductive. In Actes de l'université d'été du CIFEN, Liège, août 2001, pp. 10-18.

Vygotski, L.S. (1930/1983). Immaginazione e creatività nell'età infantile. Paideia, Italy : Editori Riuniti.

Vygotski, L.S. (1978). Mind in society. The development of higher psychological processes. Cambridge: MA: Harvard University Press.

\section{RESUME}

L'activité narrative forme un registre de l'activité plus important que l'attention que l'on a bien voulu lui donner jusqu'ici. En ergonomie, l'importance du récit commence à faire son chemin dans bon nombre d'études. Notre angle d'étude envisage l'activité narrative d'enfants guidée par des adultes en situation pédagogique, et ce, en mobilisant de façon complémentaire la théorie instrumentale de Rabardel et le cycle de l'activité créatrice de l'imagination de Vygotski. Ces deux cadres théoriques permettent d'éclairer les dimensions multi instrumentée et créative de cette activité. Ces allers-retours entre analyse en situation et concepts nourrissent un modèle de l'activité narrative (NAM) qui comprend quatre phases principales, l'exploration, l'inspiration, la production et le partage. L'activité narrative est pour le sujet une façon de développer ses ressources créatives. Pour y contribuer, les enseignants opèrent à deux niveaux. D'un côté, ils aident les enfants à opérer des genèses instrumentales à un niveau fonctionnel et sémantique. Il y a à ce stade tout à la fois un travail sur l'ouverture des ressources intérieures et sur les variations que le sujet peut entretenir avec son environnement. Plusieurs activités y contribuent, sensorielles, descriptives et manipulatoires. D'un autre côté, à l'aide de multiples médias, les enseignants engagent les enfants à constituer une sorte de «laboratoire intérieur». Les enfants sont invités à porter attention aux variations des outils du réel qu'ils expérimentent et à écouter les échos qu'ils provoquent en eux. Ces médiations interpersonnelles réflexives consistent à ouvrir la palette des ressources du sujet, à élargir les possibilités de dissociations et d'associations et à générer des matériaux qui seront utiles pour la production narrative.

\section{MOTS CLEFS}

Activité narrative, créativité, instruments, médiations, situations pédagogiques. 


\section{REFERENCEMENT}

Decortis, F. (2013). L'activité narrative dans ses dimensions multi instrumentée et créative en situation pédagogique. Activités, 10(1), 3-30, http://www.activites.org/v10n1/v10n1.pdf

Article soumis le 5 septembre 2011, accepté pour publication le 26 septembre 2012. 